\title{
Computation of resonance frequencies for Maxwell equations in non smooth domains
}

\author{
Martin Costabel, Monique Dauge \\ IRMAR, Université de Rennes 1, Campus de Beaulieu, 35042 REnNES, FrANCE \\ dauge@univ-rennes1. fr, URL: \\ http://www. maths. univ-rennes1.fr/ dauge
}

Summary. We address the computation by finite elements of the non-zero eigenvalues of the (curl, curl) bilinear form with perfect conductor boundary conditions in a polyhedral cavity. One encounters two main difficulties: (i) The infinite dimensional kernel of this bilinear form (the gradient fields), (ii) The unbounded singularities of the eigen-fields near corners and edges of the cavity. We first list possible variational spaces with their functional properties and provide a short description of the edge and corner singularities. Then we address different formulations using a Galerkin approximation by edge elements or nodal elements.

After a presentation of edge elements, we concentrate on the functional issues connected with the use of nodal elements. In the framework of conforming methods, nodal elements are mandatory if one regularizes the bilinear form (curl, curl) in order to get rid of the gradient fields. A plain regularization with the (div, div) bilinear form converges to a wrong solution if the domain has reentrant edges or corners. But remedies do exist. We will present the method of addition of singular functions, and the method of regularization with weight, where the (div, div) bilinear form is modified by the introduction of a weight which can be taken as the distance to reentrant edges or corners.

\section{Introduction}

Computing Maxwell eigenfrequencies has been an interesting challenge for the numerical analysis community for many years. Besides its many obvious and very important practical applications, ranging from signal processing over heart and brain biology to nuclear fusion, the Maxwell eigenvalue problem has been attractive because of some mathematical features that set it apart from the standard fields of elliptic eigenvalue problems.

There is, on one hand, its simplicity as one of the very basic problems in partial differential equations. On this basic level there is the relation between the Maxwell equations and the de Rham complex and algebraic topology. In the construction and analysis of special families of finite elements, based on Nédélec's edge elements or "Whitney elements", this relation plays an important role. Major progress has been made in the theory of these special elements in recent years and many questions have found satisfactory answers, but some questions concerning the approximation of the eigenvalue problem remain open.

On the other hand, the Maxwell eigenvalue problem has several rather irritating peculiarities: 
(i) The gauge invariance allows for many different variational formulations. The simplest of these are non-elliptic, have a non-empty essential spectrum, and the energy space is not compactly embedded into $L^{2}$. The effect is that straightforward discretizations do not generally give any useful approximation of the eigenvalues. Some Galerkin schemes may produce convergence of the numerical eigenvalues, but the limits may contain, in addition to the exact eigenvalues, extra spurious numbers. In other cases, the eigenvalues may converge to the exact values, but their multiplicities may be wrong.

(ii) More elaborate variational formulations can be constructed to avoid the problems coming from the infinite-dimensional eigenspace associated with zero frequency, i.e. with electro- or magnetostatic fields. If these formulations involve nonconforming or mixed finite element methods, then the error analysis of the eigenvalue problem is rather difficult, involves conditions whose range of validity is not yet fully understood, and is still incomplete in some important practical situations.

(iii) Another class of variational formulations that recover ellipticity is based on regularization or penalization. Whereas the error analysis of Galerkin approximations can be standard and simple in such cases, it is the equivalence between the original Maxwell eigenvalue problem and the regularized variational formulation that can lead to serious problems here. There are always spurious eigenvalues even for the continuous formulation, coming from some auxiliary problem, and some care has to be taken in the approximation procedure to separate the true Maxwell eigenvalues from the spurious eigenvalues. More seriously, on non-smooth domains one can have a situation where the numerical eigenvalues, although converging as the number of degrees of freedom is increased, converge to the spectrum of an entirely different problem. In other cases, it may be possible to prove convergence of the numerical eigenvalues to the correct values, but the observed convergence rate is extremely slow so the method is practically useless.

(iv) Near edges and corners of conducting bodies, electromagnetic fields tend to infinity. This has obvious implications for their numerical approximation, requiring strong mesh refinements, high degree polynomials or special singular trial functions. What is worse is that for some perfectly natural variational formulations and their finite element discretization, these singularities are not just difficult to approximate, but are impossible to approximate. Typically, the eigenmode associated with the lowest non-zero eigenfrequency exhibits these strong singularities, and therefore the approximation of this principal eigenfrequency using a standard regularized formulation discretized by conforming finite elements, can be impossible. Recently a solution to this problem has been found in the weighted regularization method.

In this article, we will explain and illustrate these interesting phenomena in detail. We give descriptions of the corner and edge singularities of the Maxwell eigenfunctions, based on those of the solutions of Helmholtz boundary value problems. Then we discuss different possibilities for choosing variational formulations and associate function spaces and explain in particular the phenomenon of non-density of smooth functions. The numerical results, even in the simplest case of a square, show 
very clearly the necessity of choosing the right variational formulation and the right finite-dimensional space of trial functions.

\section{Acknowledgements}

The authors are glad to thank

Mark AINSWORTH for his editorial initiative and his careful proof reading,

Thomas APEL for the computation of three-dimensional singularity exponents, Daniele BOFFI for numerical results with edge elements and for his advice, Peter MONK for discussions and proof reading.

\section{Contents}

1. Maxwell resonance frequencies

2. Maxwell spaces and density properties

3. Singular functions of the Dirichlet Laplace operator

4. Maxwell singular functions

5. A question and two bad answers for the Galerkin discretization of our problem

6. Mimicking the kernel: edge elements

7. Going back to nodal elements: the plain regularization

8. Plain Regularization with a Singular Function Method

9. The Weighted Regularization Method

10. Comparison between methods

\section{Maxwell resonance frequencies}

Let $\Omega$ be a bounded three-dimensional domain filled with a dielectric material of permittivity $\varepsilon$ and permeability $\mu$. The electromagnetic resonance frequencies of $\Omega$ are the numbers $\omega>0$ such that there exists an electromagnetic field $(\mathbf{E}, \mathbf{H}) \neq 0$ satisfying the equations

$$
\operatorname{curl} \mathbf{E}-\mathrm{i} \omega \mu \mathbf{H}=0 \quad \text { and } \quad \operatorname{curl} \mathbf{H}+\mathrm{i} \omega \varepsilon \mathbf{E}=0 \quad \text { in } \quad \Omega \text {. }
$$

As $\omega$ is supposed to be non-zero, taking the divergence of these two equations we obtain

$$
\operatorname{div} \varepsilon \mathbf{E}=0 \quad \text { and } \quad \operatorname{div} \mu \mathbf{H}=0 .
$$

We assume the perfect conductor boundary conditions ${ }^{1}$, that is, denoting by $\mathbf{n}$ the outer unit normal on $\partial \Omega$ :

\footnotetext{
${ }^{1}$ One could also consider impedance boundary conditions

$$
\mathbf{n} \times \mathbf{H}-\lambda(\mathbf{n} \times \mathbf{E}) \times \mathbf{n}=0 .
$$

Although the regularity of the eigenmodes would be the same as with perfect conductor boundary conditions, certain approximation properties would be, in principle, better. We will discuss this briefly later.
} 


$$
\mathbf{E} \times \mathbf{n}=0 \quad \text { and } \quad \mathbf{H} \cdot \mathbf{n}=0 \quad \text { on } \quad \partial \Omega .
$$

In this paper we discuss variational methods to solve (1.1)-(1.3) with a special emphasis on the situation where $\Omega$ is not smooth. As a standard model for this, we assume that $\Omega$ is a polyhedron, that is any domain whose boundary $\partial \Omega$ is a finite union of plane faces (which are thus polygonal). Concentrating on the problems posed by the singularities of the boundary of $\Omega$, we assume that the dielectric material filling $\Omega$ is homogeneous and isotropic, that is, after a possible change of unknowns $\varepsilon=\mu=1$. We will address generalizations of this model situation in remarks or footnotes.

Recapitulating, we are looking for non-trivial solutions of the system

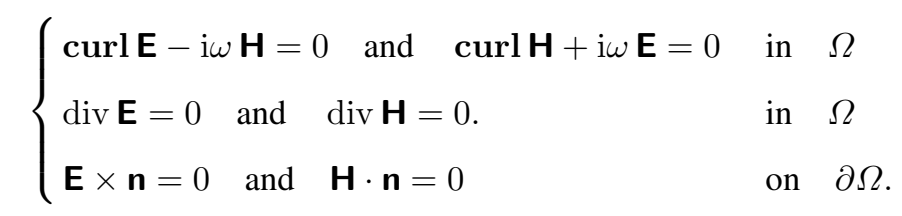

Note that, besides all non-zero $\omega$ solving (1.1),(1.3), a finite-dimensional kernel (solutions for $\omega=0$ ) will appear in (1.4) if (and only if) the topology of $\Omega$ is not trivial (i.e. if $\Omega$ is not simply connected, or $\partial \Omega$ is not connected).

\section{Maxwell spaces and density properties}

As usual in papers devoted to Maxwell equations, we will recall or introduce a number of functional spaces. We will not use all of them. But the interesting point is to study the density properties of smooth functions. In general everything goes as expected, except for a couple of spaces (the "bad" ones). In this section only, we assume for simplicity that $\Omega$ is a Lipschitz polyhedron.

\section{A A collection of spaces}

Considering system (1.4), it is natural to assume that $\mathbf{E}, \mathbf{H}$ belong to $H(\mathbf{c u r l}, \Omega)$, the space of $L^{2}(\Omega)^{3}$ fields with curl in $L^{2}(\Omega)^{3}$. As their divergence vanishes, the fields $\mathbf{E}$ and $\mathbf{H}$ also belong to $H(\operatorname{div}, \Omega)$, the space of $L^{2}(\Omega)^{3}$ fields with div in $L^{2}(\Omega)$. Since the following identities hold

$$
\begin{gathered}
\forall \mathbf{E} \in H(\operatorname{curl}, \Omega), \forall \mathbf{v} \in H^{1}(\Omega)^{3}:\langle\mathbf{E} \times \mathbf{n}, \mathbf{v}\rangle_{\partial \Omega}=\int_{\Omega} \mathbf{E} \cdot \operatorname{curl} \mathbf{v}-\operatorname{curl} \mathbf{E} \cdot \mathbf{v}, \\
\forall \mathbf{E} \in H(\operatorname{div}, \Omega), \forall \varphi \in H^{1}(\Omega):\langle\mathbf{H} \cdot \mathbf{n}, \varphi\rangle_{\partial \Omega}=\int_{\Omega} \operatorname{div} \mathbf{H} \varphi+\mathbf{H} \cdot \operatorname{grad} \varphi
\end{gathered}
$$

the tangential trace $\mathbf{E} \times \mathbf{n}$ makes sense in $H^{-1 / 2}(\partial \Omega)^{3}$ and the normal trace in $H^{-1 / 2}(\partial \Omega)$, whence the possibility of defining $H_{0}(\operatorname{curl} ; \Omega)$ as the subspace of $H(\operatorname{curl} ; \Omega)$ with zero tangential traces and $H_{0}(\operatorname{div} ; \Omega)$ as the subspace of $H(\operatorname{div} ; \Omega)$ with zero normal traces. 
For impedance conditions (1.3b), the above spaces have to be enlarged to

$$
\begin{aligned}
H\left(\operatorname{curl}, \Omega ; T L^{2}\right) & =\left\{\mathbf{u} \in H(\operatorname{curl}, \Omega) ; \quad \mathbf{u} \times\left.\mathbf{n}\right|_{\partial \Omega} \in L^{2}(\partial \Omega)^{3}\right\} \\
H\left(\operatorname{div}, \Omega ; T L^{2}\right) & =\left\{\mathbf{u} \in H(\operatorname{div}, \Omega) ;\left.\quad \mathbf{u} \cdot \mathbf{n}\right|_{\partial \Omega} \in L^{2}(\partial \Omega)\right\}
\end{aligned}
$$

where the symbol $T L^{2}$ means that the corresponding traces belong to $L^{2}$.

Finally we introduce the combined spaces (which are suitable for "regularized" formulations)

$$
\begin{array}{rll}
X_{N}=H_{0}(\text { curl }) \cap H(\operatorname{div}) & \text { and } & X_{T}=H(\text { curl }) \cap H_{0}(\operatorname{div}) \\
W_{N}=H\left(\text { curl } ; T L^{2}\right) \cap H(\operatorname{div}) & \text { and } & W_{T}=H(\text { curl }) \cap H\left(\operatorname{div} ; T L^{2}\right)
\end{array}
$$

\section{B Density of smooth functions}

The following result describes the "good" spaces where density holds:

Theorem 2.1.

$C^{\infty}(\bar{\Omega})^{3}$ is dense in $H(\operatorname{curl}, \Omega)$ and $C_{0}^{\infty}(\Omega)^{3}$ is dense in $H_{0}(\operatorname{curl}, \Omega)$, [45].

$C^{\infty}(\bar{\Omega})^{3}$ is dense in $H\left(\operatorname{curl}, \Omega ; T L^{2}\right)$, [7].

$C^{\infty}(\bar{\Omega})^{3}$ is dense in $H(\operatorname{div}, \Omega)$ and $C_{0}^{\infty}(\Omega)^{3}$ is dense in $H_{0}(\operatorname{div}, \Omega)$, [45].

$C^{\infty}(\bar{\Omega})^{3}$ is dense in $H\left(\operatorname{curl}, \Omega ; T L^{2}\right)$, [24].

$H_{0}($ curl $) \cap H_{0}(\operatorname{div})=H_{0}^{1}(\Omega)^{3}$.

The spaces $W_{N}$ and $W_{T}$ coincide and $C^{\infty}(\bar{\Omega})^{3}$ is dense in $W_{N}=W_{T},[22,25]$.

However, there are certain "bad" spaces where the closure of smooth functions stops somewhere inbetween. Let the spaces of smooth fields satisfying the zero tangential and normal trace condition be denoted by $C_{N}^{\infty}(\Omega)$ and $C_{T}^{\infty}(\Omega)$ respectively, then:

Theorem 2.2. [23, 26, 19] The closure of $C_{N}^{\infty}$ in $X_{N}$ is $H_{N}:=H^{1}(\Omega)^{3} \cap X_{N}$.

The closure of $C_{T}^{\infty}$ in $X_{T}$ is $H_{T}:=H^{1}(\Omega)^{3} \cap X_{T}$.

The possible difference between $X_{N}$ and $H_{N}$, or $X_{T}$ and $H_{T}$, is fully spanned by gradients: we note that $\operatorname{grad} \varphi \in X_{N}$ if and only if $\varphi$ belongs to the domain of the Dirichlet Laplacian

$$
D\left(\Delta^{\mathrm{Dir}}\right)=\left\{\varphi \in H_{0}^{1}(\Omega) ; \quad \Delta \varphi \in L^{2}(\Omega)\right\}
$$

and $\operatorname{grad} \varphi \in X_{T}$ if and only if $\varphi \in D\left(\Delta^{\mathrm{Neu}}\right)$, the domain of the Neumann Laplacian. In [9], it is shown that:

$$
X_{N}=H_{N}+\operatorname{grad}\left(D\left(\Delta^{\mathrm{Dir}}\right)\right) \quad \text { and } \quad X_{T}=H_{T}+\operatorname{grad}\left(D\left(\Delta^{\mathrm{Neu}}\right)\right) .
$$

If $\Omega$ is convex, then it is known [46] that the domains $D\left(\Delta^{\mathrm{Dir}}\right)$ and $D\left(\Delta^{\mathrm{Neu}}\right)$ are contained in $H^{2}(\Omega)$, but if not, then neither $D\left(\Delta^{\mathrm{Dir}}\right)$ nor $D\left(\Delta^{\mathrm{Neu}}\right)$ are contained in $H^{2}(\Omega)$. 
Decompositions of the type (2.1) have been studied in $[9,10,42]$ for various non-smooth domains. For the decomposition of $X_{N}, \Omega$ can be any Lipschitz domain and it can have cuts or "screen" parts. For the decomposition of $X_{T}$, more regularity is needed: Piecewise $C^{\alpha}$ with $\alpha>\frac{3}{2}$ will do, but in [42] is given an example of a $C^{3 / 2}$ domain (without any edges and corners) where no such decomposition exists.

\section{Singular functions of the Dirichlet Laplace operator}

In order to investigate the decompositions (2.1) for non-convex polyhedra and to prepare for a description of the non-regular parts in the solutions of (1.4), we give some information on the structure of the singular functions of the Dirichlet problem $\Delta^{\text {Dir }}$ for the Laplacian on $\Omega$ :

$$
\Delta \varphi=g \quad \text { in } \quad \Omega, \quad \text { and } \quad g \in H_{0}^{1}(\Omega) .
$$

Let us denote the set of the edges e by $\mathcal{E}$ of $\Omega$ and the set of its corners $\mathbf{c}$ by $\mathcal{C}$.

Associated with each edge or corner is: (i) a local system of coordinates, (ii) a countable set of singular functions, and (iii) corresponding templates for singular parts - here we will only mention the singular parts which are not contained in $H^{2}(\Omega)$.

\section{A For an edge $e \in \mathcal{E}$}

(i) In a neighbourhood of $\mathbf{e}, \Omega$ coincides with a dihedron of the form $\Gamma_{\mathbf{e}} \times \mathbb{R}$ where $\Gamma_{\mathbf{e}}$ is a plane sector with angle $\omega_{\mathbf{e}}$. A local system of cylindrical coordinates $(r, \theta, z)$ depending on $\mathbf{e}$ is introduced, where $r$ denotes the distance to the edge, $\theta \in\left(0, \omega_{\mathbf{e}}\right)$ and $z$ denotes a Cartesian coordinate along the edge.

(ii) The singular functions $\Phi_{\mathbf{e}}^{\ell \text {,Dir }}$ are indexed by the positive integers $\ell \in \mathbb{N}$, and given explicitly by

$$
\Phi_{\mathbf{e}}^{\ell, \operatorname{Dir}}=r^{\ell \pi / \omega_{\mathbf{e}}} \sin \frac{\ell \pi \theta}{\omega_{\mathbf{e}}} .
$$

The degree of homogeneity $\ell \pi / \omega_{\mathrm{e}}$ is the singular exponent. It is called "singular" because it is a measure of the lack of regularity of $\Phi_{\mathbf{e}}^{\ell, \text { Dir }}$ : The latter belongs to $H^{1+\tau}(\Omega)$ only if $\tau<\ell \pi / \omega_{\mathrm{e}}$. This limit is sharp, except if $\ell \pi / \omega_{\mathrm{e}}$ is an integer.

(iii) Non- $H^{2}$ singular parts along e only occur if $\omega_{\mathbf{e}}>\pi$ and $\ell=1$ : The non$H^{2}$ part of a solution of problem (3.1) with smooth right hand side $g$ has the form $\gamma_{\mathbf{e}}(z) \Phi_{\mathbf{e}}^{1, \text { Dir }}(r, \theta)$ where the edge coefficient function $\gamma_{\mathbf{e}}$, defined along the edge $\mathbf{e}$, only depends on $g$.

\section{B For a corner $\mathrm{c} \in \mathcal{C}$}

(i) In a neighborhood of $\mathbf{c}, \Omega$ coincides with a cone $\Gamma_{\mathbf{c}}$. Let $(\rho, \vartheta), \rho>0, \vartheta \in \mathbb{S}^{2}$, be spherical coordinates with origin at the vertex $\mathbf{c}$. The cone $\Gamma_{\mathrm{c}}$ is characterized by its spherical section $G_{\mathrm{c}}:=\Gamma_{\mathrm{c}} \cap \mathbb{S}^{2}$.

(ii) The singular functions $\Phi_{\mathbf{c}}^{\ell, \text { Dir }}$ at the corner $\mathbf{c}$ are given by: 


$$
\Phi_{\mathbf{c}}^{\ell, \text { Dir }}=\rho^{\lambda} \phi_{\mathbf{c}}^{\ell, \text { Dir }}(\vartheta), \quad \text { with } \quad \lambda=\lambda^{\ell, \text { Dir }}=-\frac{1}{2}+\sqrt{\mu^{\ell, \text { Dir }}+\frac{1}{4}}
$$

where the $\mu^{\ell, \text { Dir }}$ are the eigenvalues of the Laplace-Beltrami Dirichlet problem on the spherical polygon $G_{\mathbf{c}}$ and $\phi_{\mathbf{c}}^{\ell, \text { Dir }}$ are its eigenvectors. The singular exponent $\lambda^{\ell, \text { Dir }}$ also measures the regularity of $\Phi_{\mathbf{c}}^{\ell \text {,Dir }}$ in the following sense: Let $\mathcal{V}_{\mathbf{c}}$ be any cone with vertex $\mathbf{c}$ which does not intersect with the edges ${ }^{2}$, then $\Phi_{\mathbf{c}}^{\ell, \text { Dir }}$ belongs to $H^{1+\tau}\left(\mathcal{V}_{\mathbf{c}}\right)$ for $\tau<\lambda^{\ell, \text { Dir }}+\frac{1}{2}$.

(iii) Non- $H^{2}$ corner contributions (as distinct from the edge contributions) appear for $\lambda^{\ell \text {,Dir }} \leq \frac{1}{2}$. In fact, a necessary (but not sufficient) condition for this is " $\Gamma_{\mathrm{c}}$ non convex and $\ell=1$ ". Then the non- $H^{2}$ corner part has the form $\gamma_{\mathbf{c}} \Phi_{\mathbf{c}}^{1, \text { Dir }}(\rho, \vartheta)$ where the corner coefficient $\gamma_{\mathrm{c}}$ is a real number only depending on $g$.

\section{C Regularity of Dirichlet solutions}

The smallest corner exponent is $\lambda_{\mathbf{c}}^{1, D i r}$ while the smallest edge exponent is $\pi / \omega_{\mathbf{e}}$. If $g$ is smooth enough, then

$$
\begin{aligned}
& \varphi \in H^{1+\tau}(\Omega), \quad \forall<\min \left\{\tau_{\mathcal{E}}, \tau_{\mathcal{C}}^{\text {Dir }}+\frac{1}{2}\right\}, \\
& \text { where } \tau_{\mathcal{E}}=\min _{\mathbf{e} \in \mathcal{E}} \frac{\pi}{\omega_{\mathbf{e}}} \text { and } \tau_{\mathcal{C}}^{\text {Dir }}=\min _{\mathbf{c} \in \mathcal{C}} \lambda_{\mathbf{c}}^{1, \text { Dir }} .
\end{aligned}
$$

As a consequence of the results given in §3.A-3.B, we can see that the implication $g \in L^{2}(\Omega) \Rightarrow u \in H^{2}(\Omega)$ holds if and only if $\Omega$ is convex. Thus when $\Omega$ is not convex, the first decomposition in equation (2.1) is not trivial.

\section{D Origin of singularities}

A common feature of the singular functions $\Phi_{\mathrm{e}}^{\ell, \text { Dir }}$ and $\Phi_{\mathrm{c}}^{\ell \text {,Dir }}$ is that they are both harmonic in $\Gamma_{\mathbf{e}}$ and $\Gamma_{\mathbf{c}}$ and satisfy zero Dirichlet conditions on $\partial \Gamma_{\mathbf{e}}$ and $\partial \Gamma_{\mathrm{c}}$ respectively. Moreover they have $H^{1}$-regularity in any bounded neighborhood of the origin. Therefore, multiplied by a suitable cut-off function, each of them yields a singular ${ }^{3}$ solution of (3.1) with smooth right hand side.

The full theory including a splitting into regular and singular parts goes back to [52] for conical points and to [35] for polyhedra. See also [55, 37, 34].

\section{E Neumann singularities}

The edge and corner singular functions of the Laplace Neumann problem $\Delta^{\mathrm{Neu}}$ are determined in the same way. The Neumann singularities are thus denoted by $\Phi_{\mathbf{e}}^{\ell, \text { Neu }}$ and $\Phi_{\mathbf{c}}^{\ell, N e u}$. The edge singular function has the form (3.2) with sin replaced with $\cos$ and the corner singular function has the form (3.3) with exponents $\lambda_{\mathbf{c}}^{\ell, N e u}$ corresponding to the non-zero Neumann eigenvalues of the Laplace-Beltrami operator on $G_{\mathrm{c}}$.

\footnotetext{
${ }^{2}$ We have introduced these cones $\mathcal{V}_{\mathbf{c}}$ because the spherical functions $\phi_{c}^{\ell, \text { Dir }}$ have themselves singular parts at the vertices $v_{\mathbf{e}}$ of $G_{\mathbf{c}}-$ with $v_{\mathbf{e}} \in \mathbf{e}$ for each edge containing $\mathbf{c}$.

${ }^{3}$ Non-smooth in Cartesian coordinates for non-integer singular exponents, and always nonsmooth in polar or spherical coordinates.
} 


\section{Maxwell singular functions}

Eliminating $\mathbf{H}$ or $\mathbf{E}$ from (1.4), we obtain the uncoupled system

$$
\begin{aligned}
& \text { curl curl } \mathbf{E}-\omega^{2} \mathbf{E}=0, \quad \operatorname{div} \mathbf{E}=0, \quad \text { in } \Omega, \quad \mathbf{E} \times \mathbf{n}=0 \text { on } \partial \Omega \\
& \text { curlcurl } \mathbf{H}-\omega^{2} \mathbf{H}=0, \quad \operatorname{div} \mathbf{H}=0, \quad \text { in } \Omega, \quad \mathbf{H} \cdot \mathbf{n}=0 \text { on } \partial \Omega \text {. }
\end{aligned}
$$

\section{A Standard singularities}

Following [27], we obtain the electric singular functions $\mathbf{E}_{\mathbf{e}}(r, \theta)$ and $\mathbf{E}_{\mathbf{c}}(\rho, \vartheta)$ of the system (4.1) by solving two-dimensional and three-dimensional versions of the system

$$
\operatorname{curlcurl} \mathbf{E}=0, \quad \operatorname{div} \mathbf{E}=0, \quad \text { and } \quad \mathbf{E} \times \mathbf{n}=0 \text { on the boundary }
$$

on $\Gamma_{\mathbf{e}} \times \mathbb{R}$ and $\Gamma_{\mathbf{c}}$ respectively, and similarly for the magnetic singular functions $\mathbf{H}_{\mathbf{e}}$ and $\mathbf{H}_{\mathrm{c}}$. In each case, we find two ${ }^{4}$ types $\mathbf{1}$ and $\mathbf{2}$ of electric singular functions: $\mathbf{E}_{\mathbf{e}}^{\ell, \mathbf{1}}$, $\mathbf{E}_{\mathbf{c}}^{\ell, 1}$ and $\mathbf{E}_{\mathrm{e}}^{\ell, 2}, \mathbf{E}_{\mathbf{c}}^{\ell, 2}$, and their magnetic counterparts.

Type 1 contains the gradients of the singular functions of $\Delta$ :

$$
\mathbf{E}_{\mathbf{e}}^{\ell, \mathbf{1}}=\left(\operatorname{grad}_{x, y} \Phi_{\mathbf{e}}^{\ell, \text { Dir }}, 0\right) \quad \text { and } \quad \mathbf{E}_{\mathbf{c}}^{\ell, \mathbf{1}}=\operatorname{grad}_{x, y, z} \Phi_{\mathbf{c}}^{\ell, \text { Dir }}
$$

(for the magnetic field, Dir is replaced with Neu) and type 2 is defined as

$$
\mathbf{E}_{\mathbf{e}}^{\ell, 2}=\left(0,0, \Phi_{\mathbf{e}}^{\ell, \text { Dir }}\right) \quad \text { and } \quad \mathbf{E}_{\mathbf{c}}^{\ell, 2}=\operatorname{grad}_{x, y, z} \Phi_{\mathbf{c}}^{\ell, \text { Neu }} \times \mathbf{x} .
$$

Here $\mathbf{x}=(x, y, z)$ always refers to local Cartesian coordinates (centered in $\mathbf{c}$, or $z$ along $\mathbf{e}$ and $(x, y)$ transverse to $\mathbf{e})$ and the components of the field $\mathbf{E}$ are written in the same system. The corresponding results for the magnetic fields are obtained by interchanging Dir and Neu.

It is obvious that the singular functions of type 1 satisfy (4.3). For type 2 , this is a consequence of the relation, valid for any scalar function $\Phi$ homogeneous of degree $\lambda$ in the cone $\Gamma_{\mathbf{c}}$ [27, Lemma 6.2]:

$$
\operatorname{curl}(\operatorname{grad} \Phi \times \mathbf{x})=(\lambda+1) \operatorname{grad} \Phi .
$$

The corresponding singular exponents are given in Table 1. Besides that, corner types $\mathbf{1}$ and $\mathbf{2}$ exchange between the electric and magnetic fields $\mathbf{E}$ and $\mathbf{H}$ solutions of (1.4): the coefficient of $\mathbf{E}_{\mathbf{c}}^{\ell, \mathbf{1}}$ in $\mathbf{E}$ is the same as the coefficient of $-\mathrm{i} k \mathbf{H}_{\mathrm{c}}^{\ell, 2}$ in $\mathbf{H}$ with $k=\omega\left(\lambda_{\mathbf{c}}^{\ell, \text { Dir }}+1\right)^{-1}$ and $\mathbf{H}_{\mathbf{c}}^{\ell, \mathbf{1}}$ corresponds to $\mathrm{i} k \mathbf{E}_{\mathbf{c}}^{\ell, 2}$.

\section{B Topological singularities}

The above collection of corner singular functions may not be complete in the situation when the spherical section $G_{\mathbf{c}}$ of the cone $\Gamma_{\mathbf{c}}$ has a non-trivial topology, namely, when its boundary is multiply connected. Let us denote the Laplace-Beltrami operator on $G_{\mathbf{c}}$ by $\Delta_{\mathbf{c}}$ and the distinct connected components of $\partial G_{\mathbf{c}}$ by $\partial_{j} G_{\mathbf{c}}$,

\footnotetext{
${ }^{4}$ In [27] three types are described. But for divergence free solutions the third type is absent.
} 


\begin{tabular}{|c|c|c|c|}
\hline $\mathbf{E}_{\mathrm{e}}^{\ell, 1}$ & $\mathbf{E}_{\mathrm{e}}^{\ell, 2}$ & $\mathbf{E}_{\mathrm{c}}^{\ell, 1}$ & $\mathbf{E}_{\mathbf{c}}^{\ell, 2}$ \\
\hline$\frac{\ell \pi}{\omega_{\mathrm{e}}}-1$ & $\frac{\ell \pi}{\omega_{\mathrm{e}}}$ & $\lambda_{\mathrm{c}}^{\ell, \text { Dir }}-1$ & $\lambda_{\mathrm{c}}^{\ell, \text { Neu }}$ \\
\hline
\end{tabular}

Table 1. Electric singular exponents of types 1 and 2

$j=0, \ldots, J$. Then the space $P_{\mathbf{c}}^{\text {Dir }}$ of functions $\phi \in H^{1}\left(G_{\mathbf{c}}\right)$ such that $\Delta_{\mathbf{c}} \phi=0$ and with constant traces $c_{j}$ on each $\partial_{j} G_{\mathbf{c}}$ has the dimension $J+1$. Then scalar functions $\Phi$, homogeneous of degree 0 , defined by

$$
\Phi(\rho, \vartheta)=\phi(\vartheta) \quad \text { in } \quad \Gamma_{\mathbf{c}}
$$

satisfy $\Delta \Phi=0$ in $\Gamma_{\mathbf{c}}$ and have constant traces on the connected components of $\partial \Gamma_{\mathbf{c}}$. Therefore the singular fields defined as

$$
\mathbf{E}_{\mathbf{c}}(\rho, \vartheta)=\operatorname{grad} \Phi \quad \text { in } \quad \Gamma_{\mathbf{c}}
$$

are curl and div free in $\Gamma_{\mathbf{c}}$ and their tangential component $\mathbf{E}_{\mathbf{c}} \times \mathbf{n}$ is zero on $\partial \Gamma_{\mathbf{c}}$. This space of dimension $J$, together with its type 2 counterpart (space of the $\mathbf{E}_{\mathbf{c}} \times$ $\mathbf{x}$ ), definitely completes ${ }^{5}$ the set of corner singular fields for the electric part of eigenfields. For the full story, see [27, §6.4].

\section{C Regularity of Maxwell eigenfields}

For the regularity statement of the Dirichlet Laplacian, we have introduced $\tau_{\mathcal{E}}$ and $\tau_{\mathcal{C}}^{\text {Dir }}$ in (3.5). We similarly define $\tau_{\mathcal{C}}^{\mathrm{Neu}}$ as the smallest of the non-zero Neumann exponents $\lambda_{\mathbf{c}}^{\mathrm{Neu}}$ over all corners. In order to take the topological singularities into account we define

$$
\widetilde{\tau}_{\mathcal{C}}^{\text {Dir }}= \begin{cases}\tau_{\mathcal{C}}^{\text {Dir }} & \text { if all } G_{\mathbf{c}} \text { have a connected boundary } \\ 0 & \text { if at least one } G_{\mathbf{c}} \text { has a multiply connected boundary }\end{cases}
$$

and similarly for $\widetilde{\tau}_{\mathcal{C}}^{\text {Dir }}$.

Theorem 4.1. Let $(\mathbf{E}, \mathbf{H})$ be solution of (1.4). Then

$$
\begin{aligned}
\mathbf{E} \in H^{\tau}(\Omega)^{3}, \quad \forall \tau<\min \left\{\tau_{\mathcal{E}}, \widetilde{\tau}_{\mathcal{C}}^{\mathrm{Dir}}+\frac{1}{2}, \widetilde{\tau}_{\mathcal{C}}^{\mathrm{Neu}}+\frac{3}{2}\right\} \\
\text { and } \quad \mathbf{H} \in H^{\tau}(\Omega)^{3}, \quad \forall \tau<\min \left\{\tau_{\mathcal{E}}, \widetilde{\tau}_{\mathcal{C}}^{\mathrm{Neu}}+\frac{1}{2}, \widetilde{\tau}_{\mathcal{C}}^{\mathrm{Dir}}+\frac{3}{2}\right\} .
\end{aligned}
$$

Thus we see that the condition $\tau_{\mathcal{E}}>1$ (i.e. all $\omega_{\mathbf{e}}<\pi$ ) is necessary to have $H^{1}(\Omega)$-regularity. As we assume that $\Omega$ is a polyhedron, this condition implies that

\footnotetext{
${ }^{5}$ Since we have no source term, we do not have to consider polynomial right hand sides for the determination of the singular function, as was done in [27].
} 
$\Omega$ is convex. Therefore all cones $\Gamma_{\mathrm{c}}$ are convex and their spherical part $G_{\mathrm{c}}$ are convex subsets of the sphere. The minimal exponents satisfy then, see [36, $\S 4]$ :

$$
\Omega \text { convex } \Longrightarrow \quad \tau_{\mathcal{C}}^{\text {Dir }} \geq \tau_{\mathcal{E}} \quad \text { and } \quad \tau_{\mathcal{C}}^{\mathrm{Neu}} \geq \frac{\sqrt{5}-1}{2}
$$

Therefore, when the polyhedron $\Omega$ is convex, E belongs to $H^{\tau}(\Omega)^{3}$ for all $\tau<$ $\min \left\{\tau_{\mathcal{E}}, 1+\sqrt{5} / 2\right\}$.

In general, as $\tau_{\mathcal{E}} \geq \frac{1}{2}$ and $\widetilde{\tau}_{\mathcal{C}}^{\text {Dir }}, \widetilde{\tau}_{\mathcal{C}}^{\mathrm{Neu}} \geq 0$, we see that in any case $\mathbf{E}, \mathbf{H} \in$ $H^{\tau}(\Omega)^{3}$ for all $\tau<\frac{1}{2}$. Moreover, if $\Omega$ is Lipschitz, all $\omega_{\mathbf{e}}$ are less than $2 \pi$ and the topological singularities are absent. Therefore $\tau_{\mathcal{E}}>\frac{1}{2}$ and $\widetilde{\tau}_{\mathcal{C}}^{\text {Dir }}, \widetilde{\tau}_{\mathcal{C}}^{\text {Neu }}>0$, which implies that $\mathbf{E}, \mathbf{H} \in H^{\frac{1}{2}+\delta}(\Omega)^{3}$ for all $\delta<\delta_{\Omega}$ for some $\delta_{\Omega}>0$.

\section{D A decomposition of electric Maxwell eigenfields}

The splitting (2.1) is non-trivial if and only if $\Omega$ is non-convex. So, let us consider a non-convex Lipschitz ${ }^{6}$ polyhedron $\Omega$. As the electric part of eigenvectors $\mathbf{E}$ belongs to $X_{N}$, we have

$$
\mathbf{E}=\mathbf{E}_{0}+\operatorname{grad} \Phi \quad \text { with } \quad \mathbf{E}_{0} \in H_{N} \text { and } \Phi \in H_{0}^{1}(\Omega) \text { s.t. } \Delta \Phi \in L^{2}(\Omega) .
$$

Noting that singular fields of type $\mathbf{2}$ have positive exponents, it is possible to prove the following decomposition of the electric part $\mathbf{E}$ of any solution of (1.4):

$$
\mathbf{E}=\mathbf{E}_{0}+\operatorname{grad} \Phi: \begin{cases}\mathbf{E}_{0} \in H^{1+\tau}(\Omega)^{3}, & \forall \tau<\min \left\{\tau_{\mathcal{E}}, \tau_{\mathcal{C}}^{\mathrm{Neu}}+\frac{1}{2}, \tau_{\mathcal{C}}^{\text {Dir }}+\frac{1}{2}\right\} \\ \varphi \in H^{1+\tau}(\Omega), & \forall \tau<\min \left\{\tau_{\mathcal{E}}, \tau_{\mathcal{C}}^{\text {Dir }}+\frac{1}{2}\right\}\end{cases}
$$

Similar results hold for the magnetic field $\mathbf{H}$.

Let us emphasize that the decomposition (4.7)-(4.8) does not coincide with the Hodge decomposition of $\mathbf{E}: \mathbf{E}_{0}$ and $\operatorname{grad} \Phi$ are not orthogonal and there even exists an alternative to (4.7) as a decomposition with a singular part of the form curl $\mathbf{A}$ with a singular field $\mathbf{A} \in H_{T}$, see [27, Remark 4.10].

\section{E Non-homogeneous materials}

When the physical parameters $\varepsilon$ and $\mu$ are piecewise constant on a polyhedral partition $\left(\Omega_{j}\right)$ of $\Omega$, a similar theory applies, see [31]. Now the set $\mathcal{C}$ includes the corners of all sub-domains $\Omega_{j}$ and similarly for edges. We then still have singularities of type $\mathbf{1}$ and $\mathbf{2}$ for both $\mathbf{E}$ and $\mathbf{H}$ : but instead of being generated by the singular functions of the plain Laplace problems $\Delta^{\text {Dir }}$ and $\Delta^{\text {Neu }}$, their scalar densities are the singular functions of the operators $\Delta_{\varepsilon}^{\mathrm{Dir}}$ and $\Delta_{\mu}^{\mathrm{Neu}}$ (corresponding to the bilinear forms $\int_{\Omega} \varepsilon \operatorname{grad} u \cdot \operatorname{grad} v$ and $\int_{\Omega} \mu \operatorname{grad} u \cdot \operatorname{grad} v \operatorname{respectively).}$

The outcome can be a very low regularity for $\mathbf{E}$ or $\mathbf{H}$ : for any $\delta>0$, there exists $\varepsilon$ such that the the generic regularity of $\mathbf{E}$ is less than $H^{\delta}(\Omega)$.

\footnotetext{
${ }^{6}$ The decomposition (4.7) holds more generally when there are no topological singularities, and (4.8) holds also in the presence of topological singularities if $\tau$ is replaced by $\widetilde{\tau}$.
} 


\section{A question and two bad answers}

\section{A Typical spectral problems}

Hoping that Maxwell problems share the desirable properties of Laplace problems, we first look for a Galerkin formulation of problem (4.1), i.e. we seek a space $\mathfrak{X}$ and a bilinear form $a$ such that non-trivial solutions $(\mathbf{u}, \omega)$ of the variational problem

$$
\mathbf{u} \in \mathfrak{X}, \quad \forall \mathbf{v} \in \mathfrak{X}, \quad a(\mathbf{u}, \mathbf{v})=\omega^{2} \int_{\Omega} \mathbf{u} \cdot \mathbf{v} \mathrm{d} \mathbf{x}
$$

coincide with non-trivial solutions $(\mathbf{E}, \omega) \in H(\mathbf{c u r l}, \Omega) \times \mathbb{R}$ of problem (4.1). Moreover, if we discretize the above problem using finite element subspaces $\mathfrak{X}_{h}$ of $\mathfrak{X}$

$$
\mathbf{u}_{h} \in \mathfrak{X}_{h}, \quad \forall \mathbf{v}_{h} \in \mathfrak{X}_{h}, \quad a\left(\mathbf{u}_{h}, \mathbf{v}_{h}\right)=\omega_{h}^{2} \int_{\Omega} \mathbf{u}_{h} \cdot \mathbf{v}_{h} \mathrm{~d} \mathbf{x}
$$

then we require that $\omega_{h} \rightarrow \omega$ in the sense that the $k$-th non-zero eigenfrequency $\omega_{h, k}$ of $\left(\mathfrak{P}_{h}\right)$ converges to the $k$-th non-zero eigenfrequency $\omega_{k}$ of $(\mathfrak{P})$.

\section{B The minimal space is not a good choice}

The minimal space $\mathfrak{X}$ which one could take is $H_{0}(\mathbf{c u r l}) \cap H($ div $; 0)$ i.e. the space of divergence free fields in $H_{0}$ (curl). The bilinear form $a$ is then taken as the curl bilinear form $a_{0}$ :

$$
a_{0}(\mathbf{u}, \mathbf{v})=\int_{\Omega} \operatorname{curl} \mathbf{u} \cdot \operatorname{curl} \mathbf{v} \mathrm{d} \mathbf{x} .
$$

With this choice of $\mathfrak{X}$ and $a$, the continuous Problem $(\mathfrak{P})$ has exactly the Maxwell electric eigenmodes as solutions.

However any finite element space $\mathfrak{X}_{h}$ (if it exists) contained in the space $\mathfrak{X}$ would be curl and div conforming. Therefore the tangential and normal jumps of any $\mathbf{u}_{h} \in \mathfrak{X}_{h}$ across neighboring elements of the mesh will be zero, which implies that the piecewise polynomial $\mathbf{u}_{h}$ is continuous on $\bar{\Omega}$, thus grad conforming. As a consequence any $\mathbf{u}_{h}$ is contained in $H_{N}$, the subspace of $H^{1}$ fields in $X_{N}=$ $H_{0}($ curl $) \cap H($ div $)$.

Although this seems harmless ${ }^{7}$ for convex domains for which $H_{N}=X_{N}$, this fact is a real obstruction to the convergence $\omega_{h, k} \rightarrow \omega_{k}$ for a general non-convex polyhedra, since $H_{N}$ is closed in $X_{N}$, (cf Theorem 2.2), and $H_{N} \neq X_{N}$, (see (2.1), (3.4)).

\section{C The maximal space is not a good choice}

The maximal space one could take is $\mathfrak{X}=H_{0}($ curl $)$. The form $a$ is still the curl bilinear form $a_{0}$ (5.1). The non-zero eigenfrequencies of the continuous problem $(\mathfrak{P})$ are then exactly the Maxwell eigenfrequencies.

\footnotetext{
${ }^{7}$ For edge angles $\omega_{\mathrm{e}}$ close to $\pi$, the extra regularity beyond $H^{1}(\Omega)$ is very low, compare with Theorem 4.1.
} 


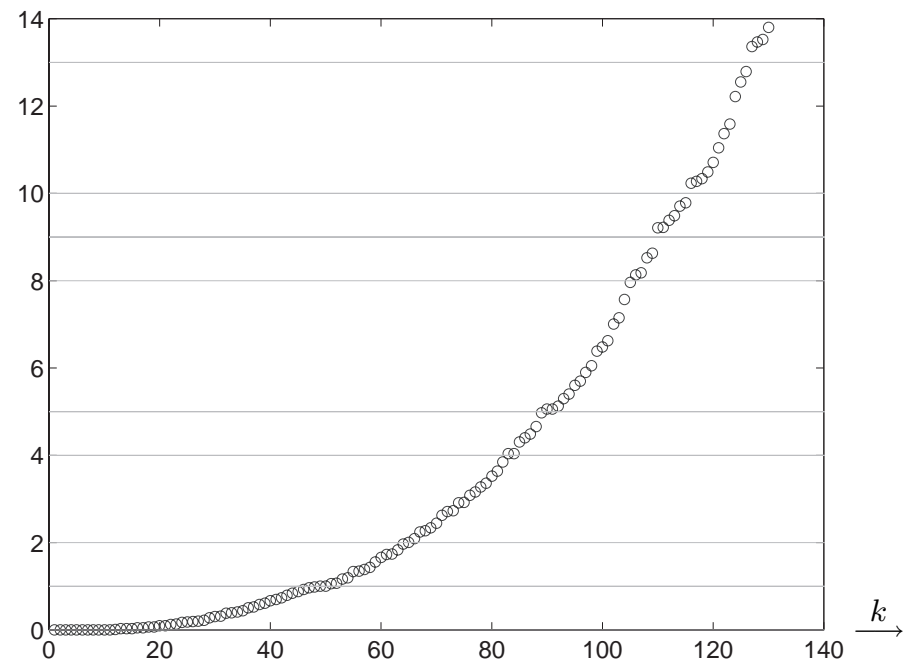

Fig. 1. Nodal triangles $\left(15\right.$ nodes per side, $\left.\mathbb{P}_{1}\right)$ [18]. Eigenvalue $\omega_{k}$ vs rank $k$.

However now $(\mathfrak{P})$ has also the infinite dimensional kernel

$$
K=\left\{u \in H_{0}(\operatorname{curl}), \quad \operatorname{curl} u=0\right\},
$$

and we note that

$$
K \subset\left\{u=\operatorname{grad} p, \quad p \in H_{0}^{1}(\Omega)\right\}
$$

(equality holds if $\Omega$ is simply connected).

The interesting spectrum lies between the two "points" 0 and $+\infty$ of the essential spectrum $^{8}$ of $(\mathfrak{P})$. The general results $[39,40]$ for the approximation of the discrete spectrum in presence of essential spectrum do not apply here. This fact has been investigated from the theoretical and computational point of view in $[14,18]$.

Here we give a simple illustration. The domain taken to be the two-dimensional ${ }^{9}$ square $[0, \pi]^{2}$. We compute the eigenvalues of the bilinear form $a_{0}$ on different discretizations of the space $H_{0}$ (curl) and we plot them in the following way: The eigenvalues are sorted by increasing order, the abscissa is the rank of the eigenvalue $\omega^{2}$, the ordinate is its value, which is marked by a circle. The horizontal lines indicate the exact values of the non-zero spectrum, which is, repeated according to multiplicity in the region $\omega^{2}<14$, given by:

$$
\left[\begin{array}{lllllllllllllll}
1 & 1 & 2 & 4 & 4 & 5 & 5 & 8 & 9 & 9 & 10 & 10 & 13 & 13
\end{array}\right]
$$

\footnotetext{
${ }^{8}$ Both points are accumulation points of the spectrum.

${ }^{9}$ In principle the computation in the 2D case has no "physical" interest since the electric Maxwell eigenvalues coincide with the Neumann eigenvalues of the Laplacian, the curl of the Laplace eigenvectors being the electric Maxwell eigenvectors. Nevertheless, a priori knowledge of eigenpairs is very valuable to test any numerical method.
} 


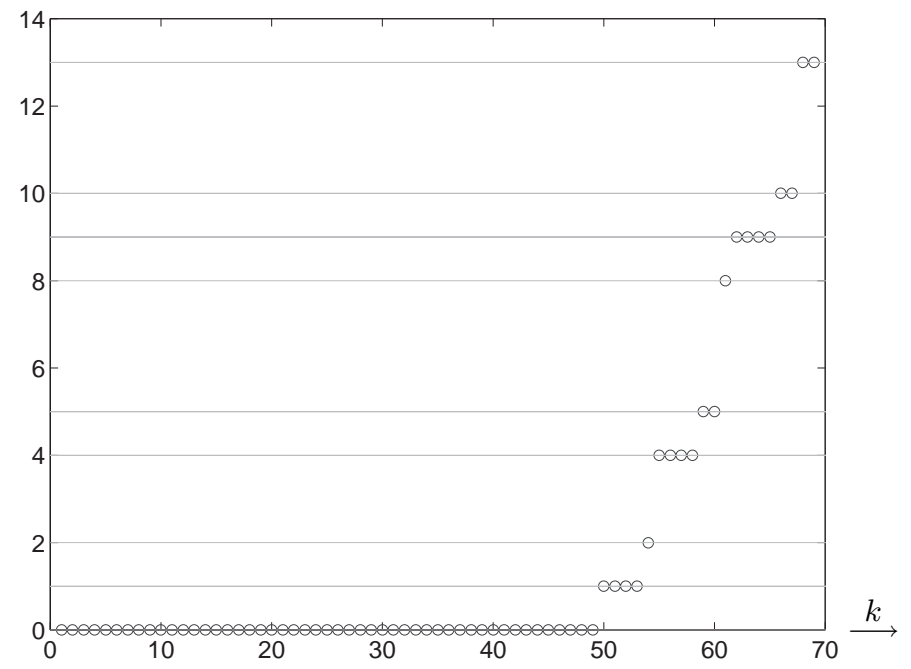

Fig. 2. One square element $\left(\mathbb{Q}_{8}\right)$. Eigenvalue $\omega_{k}$ vs rank $k$.

In Fig.1, we plot the results from [18] of a computation made with an unstructured triangular mesh containing 15 nodes per side of the square and Lagrange $\mathbb{P}_{1}$ polynomials for each of the two components of the field. The total number of unknowns is 440. The distribution of eigenvalues is very similar to that obtained for the Cosserat problem in [62] with a $p$-version code.

We have also tried the computation in our square with the $p$-version of finite elements. In Fig.2, we use only one element with tensor $\mathbb{Q}_{p}$ elements for each of the two components and the number of unknowns is $2(p+1)^{2}$. The result looks much better: we get a large kernel (computed values between $-5 \mathrm{E}-14$ and 5E-14) of dimension $(p-1)^{2}$ and the (first) correct values for the next eigenvalues, except that the multiplicity of the pure squares $(1,4,9, \ldots)$ is 4 instead of 2 as it should be (the numerical multiplicity corresponds to values that coincide very accurately with 14 common digits). The corresponding effect in a cube has been noticed by WANG \& MONK in earlier computations with $\mathbb{Q}_{4}$ elements.

The explanation of this curiosity is the following ${ }^{10}$ : If we denote the eigenpairs of the discrete 1D Dirichlet problem in $\mathbb{P}_{p}(0, \pi)$ by $\left(v_{j}^{(p)}, \lambda_{j}^{(p)}\right)$, and the Legendre polynomial of degree $p$ by $L_{p}$, we find the 4 eigenvectors

$$
\begin{aligned}
\left(v_{j}^{(p)}(y), 0\right), & \left(0, v_{j}^{(p)}(x)\right), \\
\left(L_{p}\left(\frac{2}{\pi} x-1\right) v_{j}^{(p)}(y), 0\right), & \left(0, v_{j}^{(p)}(x) L_{p}\left(\frac{2}{\pi} y-1\right)\right),
\end{aligned}
$$

associated with the eigenvalue $\lambda_{j}^{(p)}$. We can see immediately that the divergence of the first two is zero, whereas the divergence of the latter ones blows up with $p$ (the

\footnotetext{
${ }^{10}$ In the case of the square, although we expect that a similar explanation holds for the cube.
} 


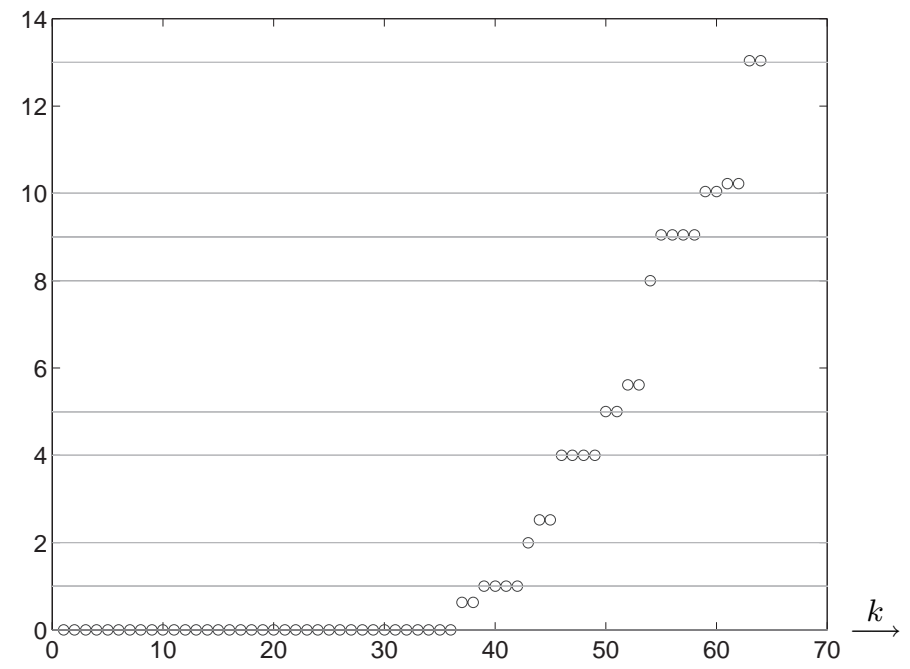

Fig. 3. Four square elements $\left(\mathbb{Q}_{4}\right)$. Eigenvalue $\omega_{k}$ vs rank $k$.

ratio of the $L^{2}$ norm of the divergence against the $H(\mathbf{c u r l})$ norm of the eigenvector behaves as $p^{3 / 2}$ ).

In Fig.3, we now use 4 elements (each side is split into two equal parts) with tensor $\mathbb{Q}_{p}$ elements, and the number of unknowns is $2(2 p+1)^{2}$. We can see the results starting to deteriorate: the kernel has dimension $4(p-1)^{2}$, the eigenvalues are correctly computed (with the same extra multiplicity) but pairs of spurious values appear also. Compare with the results obtained on a criss-cross mesh [17, §III.B].

\section{Mimicking the kernel: edge elements}

\section{A The principle}

Let us assume for simplicity that $\partial \Omega$ is connected. The principle ${ }^{11}$ is to use compatible finite element spaces and projection operators for 0 -forms (potential $p$ ) and 1-forms (electric field $\mathbf{u}$ ) according to

\begin{tabular}{|c|c|c|c|}
\hline$p$ & space $\mathrm{P}_{h} \subset H_{0}^{1}$ & grad conforming & projector $\pi_{h}$ \\
$\mathbf{u}$ & space $\mathbf{V}_{h} \subset H_{0}(\mathbf{c u r l})$ & curl conforming & projector $r_{h}$ \\
\hline
\end{tabular}

with the commuting diagram property

$$
r_{h}(\operatorname{grad} p)=\operatorname{grad}\left(\pi_{h} p\right)
$$

${ }^{11}$ For further details on edge elements, see the survey [49]. 
This leads to the following alteration of the idea of the minimal space ( $\S 5$.B). Let us denote the scalar product in $L^{2}(\Omega)$ by $\langle\cdot, \cdot\rangle$. We go back to the minimal $\mathfrak{X}$ :

$$
\mathfrak{X}=\mathfrak{X}^{\min }=\left\{\mathbf{u} \in H_{0}(\mathbf{c u r l}), \quad \forall \mathbf{v} \in K, \quad\langle\mathbf{u}, \mathbf{v}\rangle=0\right\}
$$

where $K$ is the kernel (5.2). But now we propose as discrete space:

$$
\mathfrak{X}_{h}=\mathfrak{X}_{h}^{\min }=\left\{\mathbf{u}_{h} \in \mathbf{V}_{h}, \quad \forall \mathbf{v}_{h} \in K \cap \mathbf{v}_{h}, \quad\left\langle\mathbf{u}_{h}, \mathbf{v}_{h}\right\rangle=0\right\} .
$$

Since $\partial \Omega$ is assumed to be connected, $K$ coincides with $\operatorname{grad}\left(H_{0}^{1}\right)$.

Lemma 6.1. $K \cap \mathbf{V}_{h}=\operatorname{grad}\left(\mathrm{P}_{h}\right)$.

Proof. (i) As $\mathrm{P}_{h}$ is contained in $H_{0}^{1}(\Omega)$, $\operatorname{grad}\left(\mathrm{P}_{h}\right)$ is contained in $K$. On the other hand, for any $p_{h} \in \mathrm{P}_{h},(6.1)$ gives that $\operatorname{grad}\left(p_{h}\right)=\operatorname{grad}\left(\pi_{h} p_{h}\right)=r_{h}\left(\operatorname{grad} p_{h}\right)$ which belongs to $\mathbf{V}_{h}$.

(ii) Let $\mathbf{u}_{h}$ belong to $K \cap \mathbf{V}_{h}$. As $\mathbf{u}_{h} \in K$, there exists $p \in H_{0}^{1}(\Omega)$ such that $\mathbf{u}_{h}=$ $\operatorname{grad} p$. We have, thanks to (6.1) again, $\mathbf{u}_{h}=r_{h}\left(\mathbf{u}_{h}\right)=r_{h}(\operatorname{grad} p)=\operatorname{grad}\left(\pi_{h} p\right)$. Hence $\mathbf{u}_{h} \in \operatorname{grad}\left(\mathrm{P}_{h}\right)$.

In fact, the minimal version (6.3) of the space $\mathfrak{X}_{h}$ will never be explicitly employed. But if we go back to the maximal space idea, §5.C, we take now $\mathfrak{X}_{h}$ as the whole space $\mathbf{V}_{h}$ and we are looking for the eigenpairs $\left(\mathbf{u}_{h}, \omega_{h}\right)$ satisfying

$$
\mathbf{u}_{h} \in \mathbf{V}_{h}, \quad \forall \mathbf{v}_{h} \in \mathbf{V}_{h}, \quad \int_{\Omega} \operatorname{curl} \mathbf{u}_{h}, \operatorname{curl} \mathbf{v}_{h} \mathrm{~d} \mathbf{x}=\omega_{h}^{2} \int_{\Omega} \mathbf{u}_{h} \cdot \mathbf{v}_{h} \mathrm{~d} \mathbf{x} .
$$

Then the kernel (the $\mathbf{u}_{h}$ corresponding to $\omega_{h}=0$ ) coincides with $\operatorname{grad}\left(\mathrm{P}_{h}\right)$ and the remainder of the spectrum is associated with the eigenvectors in the space $\mathfrak{X}_{h}^{\min }$. Therefore, if $\mathfrak{X}_{h}^{\min }$ is a good approximation of the space $\mathfrak{X}^{\text {min }}$, we expect (the reality is more involved, see $\S 6$.D) a good approximation of the Maxwell eigenvalues.

This is indeed illustrated, see Fig.4, by results of computations from [18] where the edge elements of order 1 on triangles are used (same square domain and same mesh as in Fig.1). The number of degrees of freedom is equal to 635. The dimension of the kernel is 194, which is the dimension of $\mathrm{P}_{h}$. We show the ranks $k$ of eigenvalues in the transition region $k \in[150,220]$ between the kernel and the remainder of the spectrum.

Note 6.2. The space $\mathfrak{X}^{\text {min }}$ is contained in the space $H$ (div; 0$)$ of divergence free fields. But, for any of the families $\left(\mathbf{V}_{h}, \mathrm{P}_{h}\right)$, there holds $\mathfrak{X}_{h}^{\min } \not \subset H$ (div; 0$)$, which means that, from the minimal space point of view, $\mathfrak{X}_{h}^{\min }$ is a non-conforming approximation. In fact $\mathfrak{X}_{h}^{\min }$ is the discrete divergence free space and a certain control of the divergence of its elements is a necessary condition to the convergence of eigenvalues as $h \rightarrow 0$. 


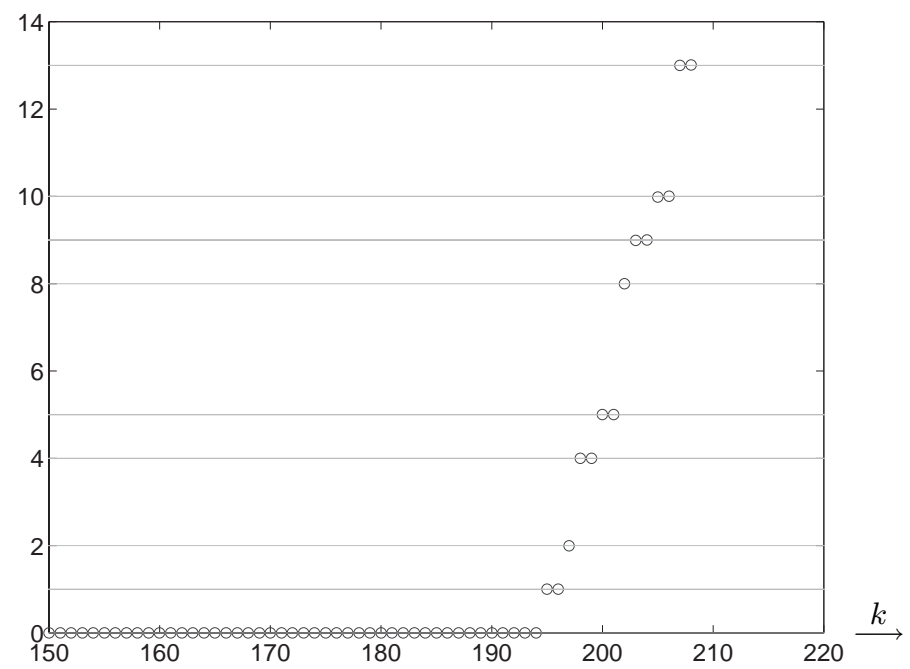

Fig. 4. Triangular edge elements (15 nodes per side, $\mathbf{P}_{1}$ ) [18]. Eigenvalue $\omega_{k}$ vs rank $k$.

\section{B Mixed formulations}

As we will see later, a much larger number of degrees of freedom is necessary in the presence of non-convex corners to obtain a decent approximation. The maximal space method obliges one to compute a large number of eigenvalues (here, nearly one third of the total spectrum in 2D) and we expect that the computational effort and the quality of approximation deteriorate for such a large number. Nevertheless see [63] for the special issue of the computation of the eigenvalues of the Laplacian by spectral methods.

To get rid of this embarrassing kernel, a mixed formulation in $\mathbf{V}_{h} \times \mathrm{P}_{h}$ is preferred, $[56,45,50,2]$ :

$\left(\mathbf{u}_{h}, p_{h}\right) \in \mathbf{V}_{h} \times \mathrm{P}_{h}, \quad \forall\left(\mathbf{v}_{h}, q_{h}\right) \in \mathbf{V}_{h} \times \mathrm{P}_{h}$,

$$
\left\{\begin{aligned}
\int_{\Omega} \operatorname{curl} \mathbf{u}_{h} \cdot \operatorname{curl} \mathbf{v}_{h}+\operatorname{grad} p_{h} \cdot \mathbf{v}_{h} & =\omega_{h}^{2} \int_{\Omega} \mathbf{u}_{h} \cdot \mathbf{v}_{h} \\
\int_{\Omega} \mathbf{u}_{h} \operatorname{grad} q_{h} & =0 .
\end{aligned}\right.
$$

We note that the continuous version of this formulation is posed in $H_{0}(\operatorname{curl}, \Omega) \times$ $H_{0}^{1}(\Omega)$, that it satisfies the inf-sup condition and that its non-zero solutions provide the Maxwell eigenvalues. The analysis of the convergence of this mixed formulation is done in $[13,14]$.

In order to have a non-degenerate saddle point problem, one can also use a regularized formulation instead, see [8], for example for given $s>0$ : 
$\left(\mathbf{u}_{h}, p_{h}\right) \in \mathbf{V}_{h} \times \mathrm{P}_{h}, \quad \forall\left(\mathbf{v}_{h}, q_{h}\right) \in \mathbf{V}_{h} \times \mathrm{P}_{h}$,

$$
\left\{\begin{aligned}
\int_{\Omega} \operatorname{curl} \mathbf{u}_{h} \cdot \operatorname{curl} \mathbf{v}_{h}+\operatorname{grad} p_{h} \cdot \mathbf{v}_{h} & =\omega_{h}^{2} \int_{\Omega} \mathbf{u}_{h} \cdot \mathbf{v}_{h} \\
\int_{\Omega} \mathbf{u}_{h} \operatorname{grad} q_{h}-\frac{1}{s} \int_{\Omega} p_{h} q_{h} & =0 .
\end{aligned}\right.
$$

The continuous version of this formulation is also posed in $H_{0}$ (curl, $\left.\Omega\right) \times H_{0}^{1}(\Omega)$. It has spurious eigenfrequencies which are exactly the values of $\omega$ such that $\omega^{2}=s \nu$, with $\nu$ any eigenvalue of the Laplace operator on $H_{0}^{1}(\Omega)$. The other eigenvalues are the Maxwell eigenvalues.

\section{C Finite element spaces}

The requirement (6.1) naturally provides subspaces of finite elements (of type $\mathfrak{X}_{h}^{\mathrm{min}}$ ) which are almost divergence-free. This is why such elements were first introduced for the discretization of Stokes and Navier-Stokes equations, see [59, 45]. Their application to Maxwell equations and their development is due to NÉDÉLEC [56, 57]. Let us very briefly describe the basic families of reference elements.

For $\mathrm{P}_{h}$ one takes as reference element the polynomial space $\mathbb{P}_{p}$ : polynomials of degree $\leq p$ in 2 variables on triangles or 3 variables on tetrahedra, or $\mathbb{Q}_{p}$ : polynomials of partial degrees $\leq p$ on the unit square $(0,1)^{2}$ or the unit cube $(0,1)^{3}$.

The corresponding reference elements for $\mathbf{V}_{h}$ are $\mathbf{P}_{p}$ and $\mathbf{Q}_{p}$ respectively, defined as follows (in 3D), with the space of homogeneous polynomials of degree $p$ denoted by $\overline{\mathbb{P}}_{p}$,

$$
\mathbf{P}_{p}=\left\{\mathbf{u}+\mathbf{v} \mid \quad \mathbf{u} \in\left(\mathbb{P}_{p-1}\right)^{3}, \quad \mathbf{v} \in\left(\overline{\mathbb{P}}_{p}\right)^{3} \text { with } \mathbf{v} \cdot \mathbf{x}=0\right\}
$$

and, with the tensor product $\mathbb{P}_{p_{1}} \otimes \mathbb{P}_{p_{2}} \otimes \mathbb{P}_{p_{3}}$ denoted by $\mathbb{Q}_{p_{1}, p_{2}, p_{3}}$,

$$
\mathbf{Q}_{p}=\left\{\mathbf{u}=\left(u_{1}, u_{2}, u_{3}\right) \mid \quad u_{1} \in \mathbb{Q}_{p-1, p, p}, \quad u_{2} \in \mathbb{Q}_{p, p-1, p}, \quad u_{3} \in \mathbb{Q}_{p, p, p-1}\right\} .
$$

It is very easy to describe the projectors $\pi_{h}$ and $r_{h}$ for first degree elements. The projector $\pi_{h}$ is the Lagrange interpolant at the nodes (vertices a of the elements), i.e. $\left(\pi_{h} p\right)(\mathbf{a})=p(\mathbf{a})$, whereas $r_{h}$ interpolates the tangential moments along the edges $\left(\mathbf{a}, \mathbf{a}^{\prime}\right)$ of the elements: $\int_{\mathbf{a}}^{\mathbf{a}^{\prime}}\left(r_{h} \mathbf{u}\right) \cdot \boldsymbol{\tau} \mathrm{d} \boldsymbol{\tau}=\int_{\mathbf{a}}^{\mathbf{a}^{\prime}} \mathbf{u} \cdot \boldsymbol{\tau} \mathrm{d} \boldsymbol{\tau}$. The chain of identities for any scalar $p$ (with enough regularity)

$$
\begin{aligned}
\int_{\mathbf{a}}^{\mathbf{a}^{\prime}}\left(r_{h} \operatorname{grad} p-\operatorname{grad} \pi_{h} p\right) \cdot \boldsymbol{\tau} \mathrm{d} \boldsymbol{\tau} & =\int_{\mathbf{a}}^{\mathbf{a}^{\prime}} \operatorname{grad}\left(p-\pi_{h} p\right) \cdot \boldsymbol{\tau} \mathrm{d} \boldsymbol{\tau} \\
& =\left(p-\pi_{h} p\right)\left(\mathbf{a}^{\prime}\right)-\left(p-\pi_{h} p\right)(\mathbf{a})=0 .
\end{aligned}
$$

proves the commuting diagram property (6.1).

The description of the general case can be carried out in the general framework of Whitney forms, see [49, $\S 3]$.

Remark 6.3. Examining the expressions (5.4)-(5.5) for the discrete eigenvectors in the standard space of $\mathbb{Q}_{p}^{2}$ vector fields on the square, we see that the two first (good) ones also belong to the edge reference space $\mathbf{Q}_{p}$, whereas the last (bad) ones do not. 


\section{D Convergence analysis}

For the abstract framework, we follow [20,21] which provides a necessary and sufficient criterion for a family of discrete spaces $\left(\mathfrak{X}_{h}\right)_{h \in \mathfrak{h}}$ to provide a "spurious-free" approximation. Here $\mathfrak{h}$ is a subset of positive numbers with the only accumulation point at zero and for any $h \in \mathfrak{h}, \mathfrak{X}_{h}$ is a subspace of $H_{0}(\operatorname{curl}, \Omega)$.

We recall that $K$ is the subspace (5.2) of the curl-free fields in $H_{0}(\operatorname{curl}, \Omega)$ and that the subspace of the fields with zero discrete divergence is, $c f(6.3)$ :

$$
\mathfrak{X}_{h}^{\min }=\left\{\mathbf{u}_{h} \in \mathfrak{X}_{h}, \quad \forall \mathbf{v}_{h} \in K \cap \mathfrak{X}_{h}, \quad\left\langle\mathbf{u}_{h}, \mathbf{v}_{h}\right\rangle=0\right\} .
$$

Finally we recall the natural norm of $H(\operatorname{curl}, \Omega)$ :

$$
\|\mathbf{v}\|_{H(\operatorname{curl}, \Omega)}=\left(\|\operatorname{curl} \mathbf{v}\|_{L^{2}(\Omega)^{3}}^{2}+\|\mathbf{v}\|_{L^{2}(\Omega)^{3}}^{2}\right)^{1 / 2} .
$$

The characteristic criteria for a spurious-free approximation are the three following conditions:

(CAS) Completeness of the Approximating Subspace

$$
\forall \mathbf{v} \in H_{0}(\mathbf{c u r l}, \Omega), \quad \lim _{h \rightarrow 0} \inf _{\mathbf{v}_{h} \in \mathfrak{X}_{h}}\left\|\mathbf{v}-\mathbf{v}_{h}\right\|_{H(\operatorname{curl}, \Omega)}=0 .
$$

(CDK) Completeness of the Discrete Kernel

$$
\forall \mathbf{v} \in H_{0}(\operatorname{curl}, \Omega) \cap K, \quad \lim _{h \rightarrow 0} \inf _{\mathbf{v}_{h} \in \mathfrak{X}_{h} \cap K}\left\|\mathbf{v}-\mathbf{v}_{h}\right\|_{H(\operatorname{curl}, \Omega)}=0 .
$$

(DCP) Discrete Compactness Property

For any sequence $\left\{\mathbf{v}_{h}\right\}_{h \in \mathfrak{h}}$ of discrete divergence free fields $\mathbf{v}_{h} \in \mathfrak{X}_{h}^{\min }$ bounded in $H(\mathbf{c u r l}, \Omega)$, there exists a subsequence $\left\{\mathbf{v}_{h}\right\}_{h \in \mathfrak{h}^{\prime}}$ and $\mathbf{w} \in L^{2}(\Omega)^{3}$ such that

$$
\lim _{h \in \mathfrak{h}^{\prime}, h \rightarrow 0}\left\|\mathbf{v}_{h}-\mathbf{w}\right\|_{L^{2}(\Omega)^{3}}=0 .
$$

Let us comment on these three conditions in the framework of general edge elements according to $\S 6$.A.

(i) Condition (CAS) is satisfied in a natural way if $\mathbf{V}_{h}$ enjoys good classical local approximation properties.

(ii) The commuting diagram property (6.1) shows that in this situation (CDK) is equivalent to the classical approximation property in $H^{1}(\Omega)$ for the nodal space family $\left(\mathrm{P}_{h}\right)$

$$
\forall \varphi \in H_{0}^{1}(\Omega), \quad \lim _{h \rightarrow 0} \inf _{\varphi_{h} \in \mathrm{P}_{h}}\left\|\varphi-\varphi_{h}\right\|_{H^{1}(\Omega)}=0 .
$$

(iii) The most original of these conditions is the Discrete Compactness Property, which was introduced by KIKUCHI [51]. It implies a certain control of the true divergence when the discrete divergence is zero. For example, (DCP) is satisfied if any sequence $\mathbf{v}_{h} \in \mathfrak{X}_{h}^{\text {min }}$ bounded in $H(\operatorname{curl}, \Omega)$ satisfies 


$$
\lim _{h \rightarrow 0}\left\|\operatorname{div} \mathbf{v}_{h}\right\|_{H^{-1}(\Omega)}=0 .
$$

The spurious-free approximation of the spectrum is defined in [20] as the conjunction of five different properties, which, when restricted to the non-zero part of the spectrum, is equivalent to the following:

Definition 6.4. Let the increasing sequence of non-zero eigenfrequencies of $(\mathfrak{P})$ be denoted by $\left(\omega_{k}\right)_{k \geq 1}$ and for any $h \in \mathfrak{h}$ denote the increasing sequence of non-zero eigenfrequencies of $\left(\mathfrak{P}_{h}\right)$ by $\left(\omega_{h, k}\right)_{k \geq 1}$. In each case the eigenfrequencies are repeated according to their multiplicities. For $h \in \mathfrak{h}$ denote the associated orthonormal systems of eigenvectors by $\left(\mathbf{u}_{h, k}\right)_{k \geq 1}$.

The problems $\left(\mathfrak{P}_{h}\right)_{h \rightarrow 0}$ are a positively spurious-free approximation of $(\mathfrak{P})$ if (i) For all $k \geq 1$,

$$
\lim _{h \rightarrow 0} \omega_{h, k}=\omega_{k},
$$

(ii) For all $k \geq 1$ and for all $h \in \mathfrak{h}$, there exists an eigenvector $\mathbf{u}_{k}^{h}$ of $(\mathfrak{P})$ associated with the eigenfrequency $\omega_{k}$ so that

$$
\lim _{h \rightarrow 0}\left\|\mathbf{u}_{k}^{h}-\mathbf{u}_{h, k}\right\|_{H(\mathbf{c u r l}, \Omega)}=0 .
$$

For our Maxwell problem, only the positive eigenvalues are meaningful. This is the reason for the introduction of a "positively spurious-free" approximation. The spurious-free approximation in [20] also requires that the discrete kernels are an approximation of the kernel of the continuous problem $(\mathfrak{P})$.

Theorem 6.5. [20] The problems $\left(\mathfrak{P}_{h}\right)_{h \rightarrow 0}$ are a positively spurious-free approximation of $(\mathfrak{P})$ if conditions (CAS), (CDK) and (DCP) are satisfied. These three conditions are also necessary if a spurious-free approximation is required.

Within the $h$-version, it is proved, see $[11,38,12,21]$, that the NÉDÉLEC families satisfy the above three conditions. But within the $p$ - and $h p$-version, no definitive result seems to be known, see [15].

Remark 6.6. In [20], a counter-example is given showing that conditions (CAS), (CDK) do not imply (DCP). Our family of tensor product spaces $\left(\mathbb{Q}_{p}^{2}\right)_{p \rightarrow \infty}$ on the square provides another counter-example: as $h=1 / p \rightarrow 0$, conditions (CAS) and (CDK) are obviously satisfied. However the growth estimate on the divergence of the eigenvectors (5.5) shows that (DCP) does not hold for this family. On the other hand, the extra multiplicity for the eigenvalues close to $1,4, \ldots$ invalidates condition (i) for the convergence of eigenvalues. A corresponding phenomenon was observed in the case of a mixed $\left(\mathbb{Q}_{1}, \mathbb{P}_{0}\right)$ formulation in [16].

\section{Going back to nodal elements: the plain regularization}

Although edge elements have their attractions, one may nevertheless prefer to try to use nodal elements for various reasons: 
- Few free source codes providing edge elements are available;

- Modifying an existing FEM code to incorporate edge elements is hard work;

- Coupling with heat or elasticity is easier when the same elements are used for each field;

- If applicable, dealing with a coercive bilinear form has advantages over a mixed formulation: monotonicity of eigenvalues, optimal convergence analysis, etc. $c f$ [5]; - Or one may simply like to develop alternative methods...

\section{A The principle}

The idea goes back to LEIS [53] and consists in blowing up the kernel $K$ by transforming the eigenvalue zero into a family of non-zero spurious eigenvalues. This is easily done, at the continuous level, by the introduction of a parameter $s>0$ and a new bilinear form - note that $a_{0}$ coincides with the curl form (5.1),

$$
a_{s}(\mathbf{u}, \mathbf{v})=\int_{\Omega} \operatorname{curl} \mathbf{u} \cdot \operatorname{curl} \mathbf{v} \mathrm{d} \mathbf{x}+s \int_{\Omega} \operatorname{div} \mathbf{u} \operatorname{div} \mathbf{v} \mathrm{d} \mathbf{x} .
$$

The variational space naturally associated with this form is $X_{N}=H_{0}($ curl $) \cap$ $H$ (div). So we have defined a new version of the generic problem $(\mathfrak{P})$ of $\S 5$.A with $\mathfrak{X}=X_{N}$ and $a=a_{s}$. For any $s>0$, the form $a_{s}$ is coercive over $X_{N}$.

Theorem 7.1. There exists a basis $\left(\mathbf{u}_{k}\right)$ of eigenvectors of problem $(\mathfrak{P})$ with $\mathfrak{X}=$ $X_{N}$ and $a=a_{s}$, associated with the increasing sequence of eigenvalues $\omega_{k}^{2}[s]$, for which the following alternative holds: For each $k>0$ we have either (i) or (ii),

(i) The pair $\left(\mathbf{u}_{k}, \omega_{k}^{2}[s]\right)$ is a Maxwell eigenpair solution of (4.1).

(ii) There exists an eigenpair $(\varphi, \nu)$ of the Dirichlet Laplacian $\Delta^{\text {Dir }}$ such that $\mathbf{u}_{k}=$ $\operatorname{grad} \varphi$ and $\omega_{k}^{2}=s \nu$.

Conversely any non-trivial solution $(\mathbf{E}, \omega)$ of (4.1) is a combination of pairs of type (i) with $\omega=\omega_{k}$.

This result is proved in [26]. As a consequence, we see that the eigenvectors do not depend on $s$ (only their ranks do) and can be organized in two families: the Maxwell eigenvectors for which the divergence part of the energy $a_{s}(\mathbf{u}, \mathbf{u})$ is zero, and the "spurious" eigenvectors for which the curl part of the energy is zero.

In the literature, this method is often referred to as a "penalty method", as opposed to the "mixed methods" based on edge elements, see $\S 6$.A. We prefer the term of "regularization", because the exact determination of a Maxwell eigenvalue does not require that $s$ tends to infinity.

When discretized using finite element subspaces $\mathfrak{X}_{h}$, the form $a_{s}$ is expected to provide two families of eigenvectors $\mathbf{u}_{h, k}$ which can be identified by the value of the ratio

$$
\tau\left(\mathbf{u}_{h}\right)=\frac{\left\|\operatorname{curl} \mathbf{u}_{h}\right\|_{L^{2}(\Omega)}^{2}}{s\left\|\operatorname{div} \mathbf{u}_{h}\right\|_{L^{2}(\Omega)}^{2}} .
$$

We expect large values of $\tau$ for the approximation of Maxwell eigenvectors and small ones for the approximation of spurious eigenvectors. 


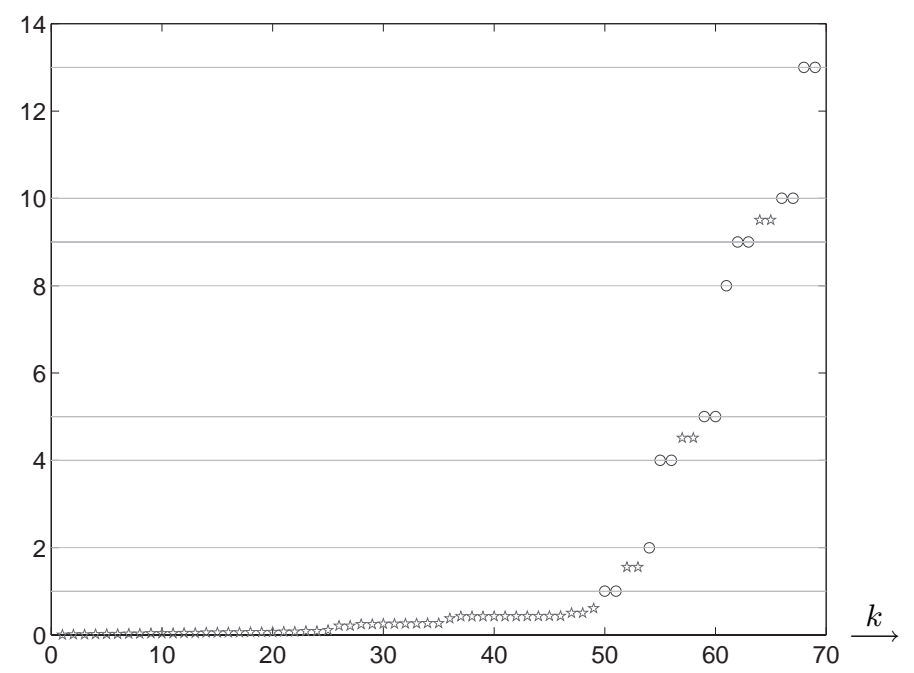

Fig. 5. One square element $\left(\mathbb{Q}_{8}\right)$ and $s=0.002$. Eigenvalue $\omega_{k}$ vs rank $k$.

\section{B In the square}

We apply this procedure on the same square domain as before ( $c f$ Figures 1-4): We compute the eigenpairs with the one-element $\mathbb{Q}_{8}$ approximation and a small value of $s=0.002$. For each eigenpair, we compute $\tau$ and mark the corresponding eigenvalue by a circle if $\tau \geq \tau_{0}$, by a "pentagram" if $\tau \leq \tau_{0}^{-1}$ and by a triangle if $\tau_{0}^{-1}<\tau<\tau_{0}$.

No triangles are observed when we choose $\tau_{0}=50$. If we compare with Fig.2, we see that the 49 zero eigenvalues rise to positive values, and that the 2 extra ones on $1,4,9, \ldots$ rise also! This is due to the large value of the divergences of the related eigenvectors, $c f$. (5.5).

We represent the eigenvalues of $a_{s}$ in a different way now. We let $s$ vary and try to follow the representatives of the two families. We do that by post-processing the results where we list for each value of $s$, the first 66 eigenvalues with the curl and divergence energies of their eigenvectors, which allows us to compute $\tau$.

We can see that the two families are easily distinguishable, the Maxwell ones are constant with respect to $s$ and provide a good approximation of the true ones (still represented by horizontal solid lines) and the spurious ones are perfectly linear with respect to $s$ (we have only plotted the first 15 lines for the sake of clarity of the figure - you see only 9 due to multiple eigenvalues). Similar computations are shown in [1, Fig. 5.2] (without postprocessing of the eigenvalues).

So, everything well in the best possible world?

\section{C In the L-shaped domain}

Let us consider the L-shaped domain $(-1,1)^{2} \backslash(-1,0)^{2}$. We use a 9 element mesh: 3 small squares of size $1 / 16$ around $(0,0)$ and the remaining "annulus" split into 


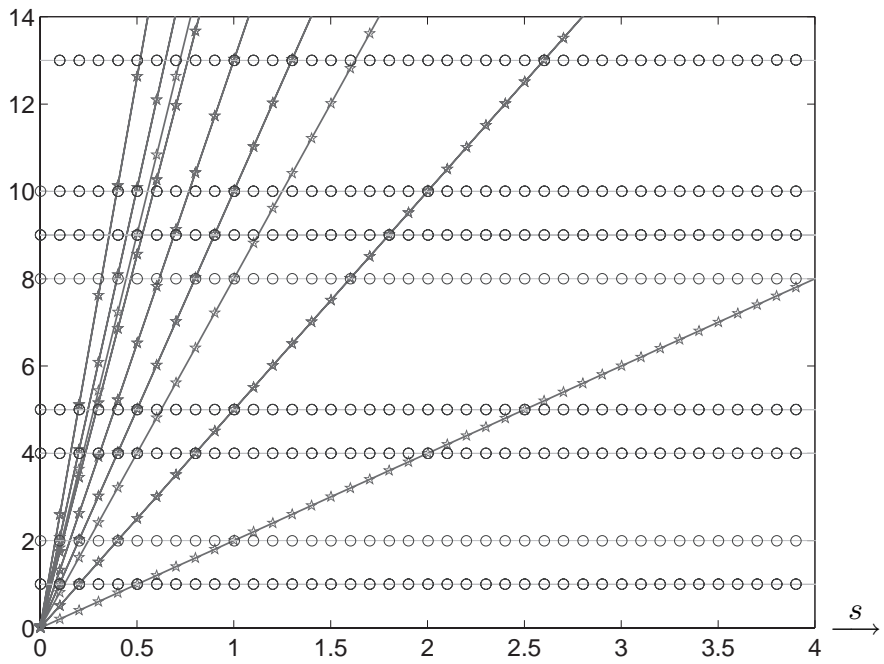

Fig. 6. One square element $\left(\mathbb{Q}_{8}\right)$ and $s=0.002 \rightarrow 4$. Eigenvalues vs parameter $s$.

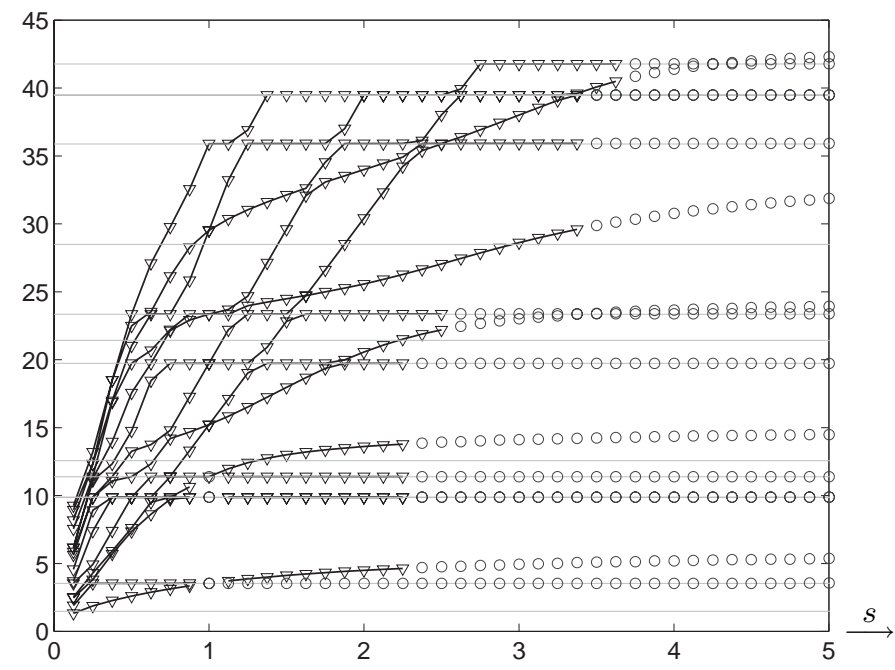

Fig. 7. L-shape $\left(9\right.$ elements $\left.\mathbb{Q}_{10}\right)$ and $s=0.125 \rightarrow 5$. Eigenvalues vs parameter $s$.

6 identical trapezia. We take $\mathbb{Q}_{10}$ polynomials on the reference element. There are 1902 degrees of freedom. We have a precise evaluation of the true solution by a computation of the eigenvalues of the scalar Neumann problem on the same domain: These values are represented as horizontal (gray) lines.

On Fig.7, we can see that the sorting criterion does not work everywhere, and that certain families of eigenvalues prefer to organize themselves on curved lines. Moreover, while certain true eigenvalues are correctly approximated for any value 
of $s$ (\# 2, 3, 4, 6, 8, 10, 11, 12, - disregarding the multiplicity) others are completely left apart $(\# 1,5,7,9)$. The reason for this is that the value of the coefficient $\gamma_{\mathbf{c}}$ of the first singularity $\operatorname{grad}\left(r^{2 / 3} \sin 2 \pi \theta / 3\right)$ : When $\gamma_{\mathbf{c}}$ is non-zero, the eigenvector does not belong to $H^{1}$ and cannot therefore be approximated.

The reason behind these odd phenomena is not a poor approximation: More refined meshes with more elements would give almost the same results. The reason is that each of these Galerkin spaces is contained in $H_{N}$ which is closed in the continuous variational space $X_{N}$, and nevertheless does not coincide with it since $\Omega$ is not convex, see Theorem 2.2 and (2.1).

In the two next sections we present two main strategies to overcome this major problem: The singular function method (SFM) and the weighted regularization method (WRM). Finally we also mention from [17] two more methods based on regularization.

\section{Plain Regularization with a Singular Function Method}

The methods based on this idea are described in the survey note [47] which also provides many references. Here we will briefly mention them, referring the reader to [47] for more details. The common idea of these methods amounts to replacing the standard finite element spaces with spaces augmented by these singular functions which cannot be approximated by the (nodal) finite element spaces.

To our knowledge, these methods have not been applied to eigenvalue problems, but insofar as they rely on a Galerkin method, they can be applied to the computation of eigenvalues as well.

They require an explicit knowledge of the singularities and, until now, they have been successfully applied only to two-dimensional problems or axisymmetric geometries (where the angular Fourier decomposition transforms the threedimensional problem into a series of problems in a meridian plane).

\section{A Singularity as a gradient}

In a $2 \mathrm{D}$ polygonal domain, one augments nodal finite element spaces by the fields

$$
\mathbf{S}_{\mathbf{c}}^{\text {Dir }}:=\operatorname{grad}\left(r^{\pi / \omega_{\mathbf{c}}} \sin \frac{\pi \theta}{\omega_{\mathbf{c}}}\right), \quad \forall \mathbf{c} \text { reentrant corner. }
$$

In order to implement the essential boundary conditions, the usual cut-off is not used (because this would generate large errors) and a discrete correction of $\mathbf{S}_{\mathrm{c}}^{\text {Dir }}$ is preferred. Instead of $\mathbf{S}_{\mathbf{c}}^{\text {Dir }}$, one considers the corrected singularities $\mathbf{E}_{\mathbf{c}, h}:=\mathbf{S}_{\mathbf{c}}^{\text {Dir }}$ $\mathbf{T}_{\mathbf{c}, h}$ where $\mathbf{T}_{\mathbf{c}, h} \in X_{h}$ is the solution of the discrete problem

$$
\forall \mathbf{v}_{h} \in X_{h, 0}, \quad a_{1}\left(\mathbf{T}_{\mathbf{c}, h}, \mathbf{v}_{h}\right)=0 \quad \text { and } \quad \mathbf{T}_{\mathbf{c}, h} \times \mathbf{n}=\mathbf{S}_{c}^{\text {Dir }} \times \mathbf{n} \text { on } \partial \Omega .
$$

Here $X_{h}$ is the full nodal vector field space and $X_{h, 0}$ is the subspace of the fields $\mathbf{v}$ which satisfy the boundary condition $\mathbf{v} \times \mathbf{n}=0$. The bilinear form $a_{1}$ is defined in (7.1) for $s=1$. For further details, see [19, 48].

A similar technique may be applied at an axisymmetric 3D conical point [43]. 


\section{B Divergence-free singularities}

In a $2 \mathrm{D}$ polygonal domain, the main singularity to be considered is

$$
\mathbf{S}_{\mathbf{c}}^{\mathrm{Neu}}:=\operatorname{curl}\left(r^{\pi / \omega_{\mathbf{c}}} \cos \frac{\pi \theta}{\omega_{\mathbf{c}}}\right), \quad \forall \mathbf{c} \text { reentrant corner, }
$$

which in fact coincides with $\mathbf{S}_{\mathbf{c}}^{\text {Dir }}$ ! The distinction lies in the correction which is adopted for $\mathbf{S}_{\mathbf{c}}^{N e u}$. Let $\Phi_{\mathbf{c}}^{\text {Neu }}$ be the Neumann singular function $r^{\pi / \omega_{\mathbf{c}}} \cos \left(\pi \theta / \omega_{\mathbf{c}}\right)$ and let us denote by $H_{\mathrm{Neu}}^{2}(\Omega)$ the subspace of functions with zero normal derivative on $\partial \Omega$. There exists a unique $\Psi_{\mathbf{c}} \in H_{\mathrm{Neu}}^{2}(\Omega)$ such that

$$
\forall \Psi \in H_{\mathrm{Neu}}^{2}(\Omega), \quad \int_{\Omega} \Delta\left(\Phi_{\mathbf{c}}^{\mathrm{Neu}}-\Psi_{\mathbf{c}}\right) \Delta \Psi=0 .
$$

Then the singularity which is added in the method is $\mathbf{S}_{\mathbf{c}}^{\mathrm{Neu}}-\operatorname{curl}\left(\Phi_{\mathbf{c}}^{\mathrm{Neu}}\right)$. This singularity is divergence-free and orthogonal to $H_{N} \cap H$ (div; 0 ). Further details are given in [4].

\section{The Weighted Regularization Method}

The singular function method appears to be difficult to analyze and implement in the case of a three-dimensional polyhedron, because of the complex structure of the infinite dimensional space of non- $H^{1}$ singular functions. The alternative to the SFM is to use the regularization together with a relaxation of the topology of the variational space. In this section we focus on the weighted regularization where the divergence part of the energy is lowered by the adjunction of a weight which tends to zero near non convex corners and edges. This idea can be compared to the technique in [58] where the contribution to the divergence integral of the elements near non convex corners is simply ignored.

\section{A The principle}

The idea and the analysis of the weighted regularization is presented in [28]. It consists of generalizing the basic regularized form (7.1) by the introduction of a weight $w$ in the divergence term, so that $a_{s}$ is now replaced by $a_{s, w}$ defined as

$$
a_{s, w}(\mathbf{u}, \mathbf{v}):=a_{0}(\mathbf{u}, \mathbf{v})+s \int_{\Omega} w(\mathbf{x})^{2} \operatorname{div} \mathbf{u} \operatorname{div} \mathbf{v} \mathrm{d} \mathbf{x},
$$

where $w>0$ is a weight. The associated variational space is now

$$
X_{N}^{w}:=H_{0}(\mathbf{c u r l}) \cap\left\{\mathbf{v} \in L^{2}(\Omega)^{3}, w \operatorname{div} \mathbf{v} \in L^{2}(\Omega)\right\} .
$$

Taking $a=a_{s, w}$ and $\mathfrak{X}=X_{N}^{w}$ yields a new version of our problem $(\mathfrak{P}), \S 5$.A:

$$
\left(\mathfrak{P}_{s, w}\right) \quad \mathbf{u} \in X_{N}^{w}, \quad \forall \mathbf{v} \in X_{N}^{w}, \quad a_{s, w}(\mathbf{u}, \mathbf{v})=\omega^{2} \int_{\Omega} \mathbf{u} \cdot \mathbf{v} \mathrm{d} \mathbf{x} .
$$

The following result generalizes Theorem 7.1 on the natural splitting of the spectrum of problem $\left(\mathfrak{P}_{s, w}\right)$. 
Theorem 9.1. Suppose that the weight $w$ ensures the following embeddings hold:

$$
L^{2}(\Omega) \subset\left\{p \in L_{\text {loc }}^{2}(\Omega) ; w p \in L^{2}(\Omega)\right\} \subset H^{-1}(\Omega) .
$$

Then there exists a basis $\left(\mathbf{u}_{k}\right)$ of eigenvectors of problem $\left(\mathfrak{P}_{s, w}\right)$ associated with the increasing sequence of eigenvalues $\omega_{k}^{2}[s, w]$, for which the following alternative holds: For each $k>0$ we have either (i) or (ii),

(i) The pair $\left(\mathbf{u}_{k}, \omega_{k}^{2}[s, w]\right)$ is a Maxwell eigenpair solution of (4.1).

(ii) There exists an eigenpair $(\varphi, \nu)$ of the Dirichlet weighted Laplacian $w \Delta(w \cdot)$ so that $\mathbf{u}_{k}=\operatorname{grad} \varphi$ and $\omega_{k}^{2}=s \nu$.

Conversely any non-trivial solution $(\mathbf{E}, \omega)$ of (4.1) is a combination of pairs of type (i) with $\omega=\omega_{k}$.

\section{B The class of weights}

We choose the weight $w$ in the form

$$
w(\mathbf{x})=d_{0}(\mathbf{x})^{\gamma} \quad \text { with } \quad d_{0}(\mathbf{x})=\operatorname{dist}\left(\mathbf{x}, \mathfrak{S}_{0}\right)
$$

where $\mathfrak{S}_{0}$ is the set of non-convex corners if $\Omega$ is a polygon, or the set of non-convex edges if $\Omega$ is a polyhedron.

Note 9.2. Of course we can replace $d_{0}$ in (9.2) by any function $\tilde{d}_{0}$ equivalent to $d_{0}$ on $\bar{\Omega}$. In polygons, the set $\mathfrak{S}_{0}$ is the union of a finite number of corner points and it is very easy to define a function $\tilde{d}_{0}$ : we can take for example the product of the distances to each corner. In polyhedra, one has to be more careful. If several nonconvex edges e have a common end point $\mathbf{c} \in \mathcal{C}$, then we cannot take $\tilde{d}_{0}$ as the product of distances to each $\mathbf{e}$. We will again comment on this choice when giving numerical results.

We have:

Lemma 9.3. Let $L_{\gamma}^{2}(\Omega)$ be the weighted space $\left\{p \in L_{\text {loc }}^{2}(\Omega) ; d_{0}^{\gamma} p \in L^{2}(\Omega)\right\}$. The embeddings (9.1): $L^{2}(\Omega) \subset L_{\gamma}^{2}(\Omega) \subset H^{-1}(\Omega)$ hold if and only if $0 \leq \gamma \leq 1$.

It is possible to prove a direct analogue of the splittings (2.1) in the setting of weighted spaces (Theorem 9.4 below). The standard Dirichlet Laplacian $\Delta^{\text {Dir }}$ has to be replaced by its weighted counterpart $\Delta_{\gamma}^{\text {Dir }}$ defined as

$$
\begin{aligned}
\Delta_{\gamma}^{\text {Dir }}: D\left(\Delta_{\gamma}^{\text {Dir }}\right):=\left\{\varphi \in H_{0}^{1}(\Omega) ; \Delta \varphi \in L_{\gamma}^{2}(\Omega)\right\} & \longrightarrow L_{\gamma}^{2}(\Omega) \\
\varphi & \longmapsto \Delta \varphi .
\end{aligned}
$$

The variational space adapted to the regularization using the weight $w=d_{0}^{\gamma}$ is given by

$$
X_{N}^{\gamma}:=H_{0}(\operatorname{curl}) \cap\left\{\mathbf{v} \in L^{2}(\Omega)^{3}, \operatorname{div} \mathbf{v} \in L_{\gamma}^{2}(\Omega)\right\}
$$

with norm

$$
\|\mathbf{v}\|_{X_{N}^{\gamma}}=\left(\|\mathbf{v}\|_{L^{2}(\Omega)}^{2}+\|\operatorname{curl} \mathbf{v}\|_{L^{2}(\Omega)}^{2}+\|\operatorname{div} \mathbf{v}\|_{L_{\gamma}^{2}(\Omega)}^{2}\right)^{1 / 2} .
$$


Theorem 9.4. [28, Th.1.2 \& 1.4] (i) Any element $\mathbf{v} \in X_{N}^{\gamma}$ can be decomposed into the sum

$$
\mathbf{v}=\mathbf{w}+\operatorname{grad} \varphi, \quad \text { with } \mathbf{w} \in H_{N} \text { and } \varphi \in D\left(\Delta_{\gamma}^{\text {Dir }}\right)
$$

such that the following estimate holds

$$
\|\mathbf{w}\|_{H^{1}(\Omega)^{3}}+\|\varphi\|_{H^{1}(\Omega)}+\|\Delta \varphi\|_{L_{\gamma}^{2}(\Omega)} \leq C\|\mathbf{v}\|_{X_{N}^{\gamma}} .
$$

(ii) If $H^{2} \cap H_{0}^{1}(\Omega)$ is dense in $D\left(\Delta_{\gamma}^{\mathrm{Dir}}\right)$, then $H_{N}$ is dense in $X_{N}^{\gamma}$.

\section{C Choosing the weight}

With the previous theorem, we do not yet know whether the introduction of a weight helps for the density of smooth functions. The answer is provided in the next statement.

Theorem 9.5. [28, Th.4.1] For any polygonal or polyhedral domain, there exists $\tau(\Omega)>0$ such that

$$
\forall \gamma, \quad 1-\tau(\Omega)<\gamma \leq 1, \quad H_{N} \text { is dense in } X_{N}^{\gamma} .
$$

Let $\tau_{\mathcal{E}}=\min _{\mathbf{e} \in \mathcal{E}}\left(\pi / \omega_{\mathbf{e}}\right)$ and let $\tau_{\mathcal{C}}^{\text {Dir }}$ be defined in (3.5), then $\tau(\Omega)$ is given by:

$$
\tau(\Omega)=\tau_{\mathcal{E}} \quad \text { in } 2 \mathrm{D} \quad \text { and } \quad \tau(\Omega)=\min \left\{\tau_{\mathcal{E}}, \tau_{\mathcal{C}}^{\text {Dir }}+\frac{1}{2}\right\} \quad \text { in 3D. }
$$

The proof of this theorem relies on (ii) of Theorem 9.4 together with a characterization of the domain of $\Delta_{\gamma}^{\text {Dir }}$ : when (9.3) holds, the domain $D\left(\Delta_{\gamma}^{\text {Dir }}\right)$ coincides with the following weighted space:

$$
V_{\gamma}^{2}(\Omega)=\left\{p \in L_{\text {loc }}^{2}(\Omega) ; d_{0}^{\gamma} d^{|\alpha|-2} \partial^{\alpha} p \in L^{2}(\Omega),|\alpha| \leq 2\right\}
$$

where $d$ is the distance to the set $\mathfrak{S}$ of all corners (in 2D) and edges (in 3D). In fact, the singularities described in $\S 3$ belong to $V_{\gamma}^{2}(\Omega)$ for all $\gamma$ satisfying condition (9.3).

\section{D Convergence of the spectrum}

Let $L_{\gamma}^{\text {Dir }}$ denote the weighted Laplacian $d^{\gamma} \Delta\left(d^{\gamma}\right.$. $)$ which is responsible for the "spurious" part of the spectrum, see (ii) in Theorem 9.1. Its spectrum $\sigma\left(L_{\gamma}^{\text {Dir }}\right)$ has the following properties:

Lemma 9.6. (i) $\sigma\left(L_{\gamma}^{\text {Dir }}\right)$ decreases as $\gamma$ increases.

(ii) There exists $\sigma_{0}>0$ such that:

$$
\forall \gamma \leq 1, \quad 0<\sigma_{0} \leq \sigma\left(L_{\gamma}^{\text {Dir }}\right) .
$$

(iii) For all $\gamma<1, L_{\gamma}^{\text {Dir }}$ has a purely discrete spectrum with accumulation at $+\infty$. (iv) For $\gamma=1, \sigma\left(L_{\gamma}^{\text {Dir }}\right)$ contains a full interval $\left[\sigma_{1},+\infty\right)$ of essential spectrum. 
Let $\left(\mathfrak{X}_{h}\right)_{h}$ be a conforming finite element approximation of $X_{N}^{\gamma}$. We can apply the result of [5] to our case. Let $\omega$ be an eigenfrequency of problem $(\mathfrak{P})$ with multiplicity $q, \omega=\omega_{\ell}=\ldots=\omega_{\ell+q-1}$, and let us denote the corresponding approximate eigenpairs by $\left(\omega_{h, k}, \mathbf{u}_{h, k}\right), k=\ell, \ldots, \ell+q-1$. We have the estimate:

$$
\left|\left(\omega_{h, k}-\omega\right) / \omega\right| \leq C \inf _{\mathbf{v}_{h} \in \mathfrak{X}_{h}}\left\|E_{\omega} \mathbf{u}_{h, k}-\mathbf{v}_{h}\right\|_{X_{N}^{\gamma}}^{2}, \quad k=\ell, \ldots, \ell+q-1 .
$$

Here $E_{\omega}$ is the orthogonal projection of $X_{N}^{\gamma}$ onto the exact eigenspace of problem $(\mathfrak{P})$ corresponding to $\omega$. Combining this with Theorem 9.1 and Lemma 9.6, we obtain:

Corollary 9.7. Let $\omega_{k}$ be the $k$-th Maxwell eigenfrequency, cf problem (4.1). Let $\gamma \in$ $[0,1]$ and $s$ be such that $\omega_{k}<s \min \sigma\left(L_{\gamma}^{\text {Dir }}\right)$. Let $\omega_{h, k}$ be the $k$-th eigenfrequency of the discrete problem $\left(\mathfrak{P}_{h}\right)$ : Find $\mathbf{u}_{h} \in \mathfrak{X}_{h}$

$$
\forall \mathbf{v}_{h} \in \mathfrak{X}_{h}, \quad a_{0}\left(\mathbf{u}_{h}, \mathbf{v}_{h}\right)+s \int_{\Omega} d_{0}(\mathbf{x})^{2 \gamma} \operatorname{div} \mathbf{u}_{h} \operatorname{div} \mathbf{v}_{h} \mathrm{~d} \mathbf{x}=\omega_{h}^{2} \int_{\Omega} \mathbf{u}_{h} \cdot \mathbf{v}_{h} \mathrm{~d} \mathbf{x} .
$$

If $S\left[\omega_{k}\right]$ denotes the unit ball of the Maxwell eigenspace associated with the eigenvalue $\omega_{k}$, then the estimate holds

$$
\left|\omega_{h, k}-\omega_{k}\right| \leq C \sup _{\mathbf{u} \in S\left[\omega_{k}\right]} \inf _{\mathbf{v}_{h} \in \mathfrak{X}_{h}}\left\|\mathbf{u}-\mathbf{v}_{h}\right\|_{X_{N}^{\gamma}}^{2} .
$$

Given suitable weight according to condition (9.3), we obtain from [28, Th.7.4] convergence rates for eigenfrequencies for the $h$-version of the finite element method with nodal elements via the weighted regularization method.

If an $h p$-version of finite elements is used (geometrical refinement of the mesh and high degree polynomials [61]), it is possible to prove that the weighted regularization method inherits the exponential convergence rates obtained for the Laplace operator, see [32] for 2D domains.

\section{E Experiments in the L-shaped domain}

We return to the example given in §7.C and illustrated in Fig.7. We can show that for such a domain the lower bound $\sigma_{0}(9.4)$ of the spurious Laplacian is $\frac{4}{9}$. We use the same 9 element mesh and the space $\mathbb{Q}_{10}$. We only change the bilinear form, using $a_{s, w}$ with $w=r^{1 / 2}$ in Fig. 8 and $w=r$ in Fig.9 instead of the plain $a_{s}$ (in this case we have $\left.d_{0}(\mathbf{x})=r\right)$. We recall that the "exact" values are computed as the Neumann eigenvalues of the Laplace operator and are represented as horizontal lines in both figures. The representation of computed eigenvalues as circles, triangles or pentagrams follow the same criteria described in $\S 7 . B$.

The improvement of the approximation when going from the plain regularization to the weighted regularization is visible (recall that the same number of unknowns (1902) is employed for the results of Fig.7 to 9): For $\gamma=\frac{1}{2}$ (recall that the infimum of admissible values for $\gamma$ is $\frac{1}{3}$ ), we see that the eigenvalues \# 1, 5, 7, 9 which were "forgotten" by the plain regularization, are now approximated - albeit less accurately than the others. 


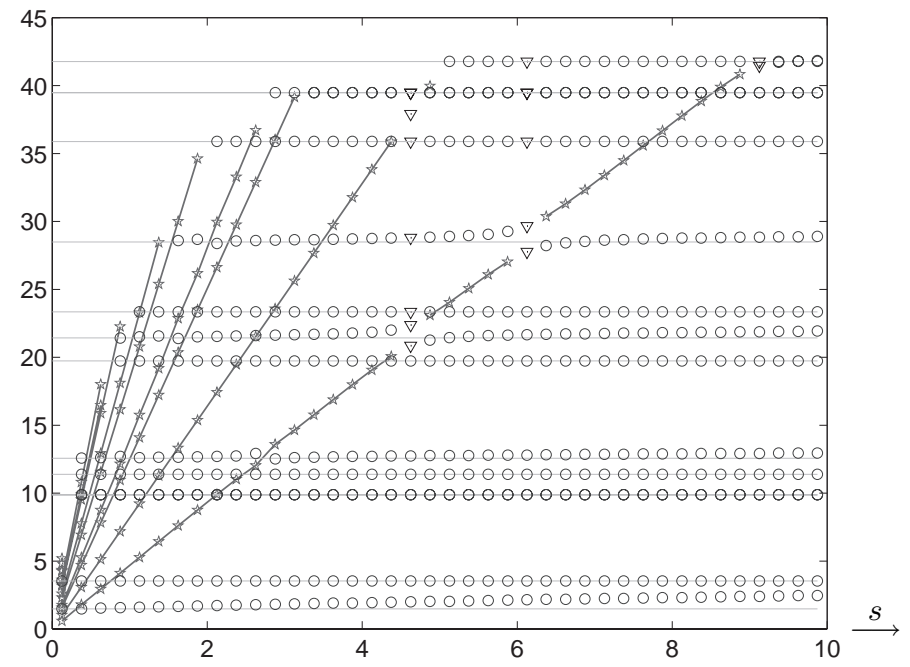

Fig. 8. L-shape, WRM with $\gamma=\frac{1}{2}$ and $s: 0.125 \rightarrow 10$. Eigenvalues vs parameter $s$.

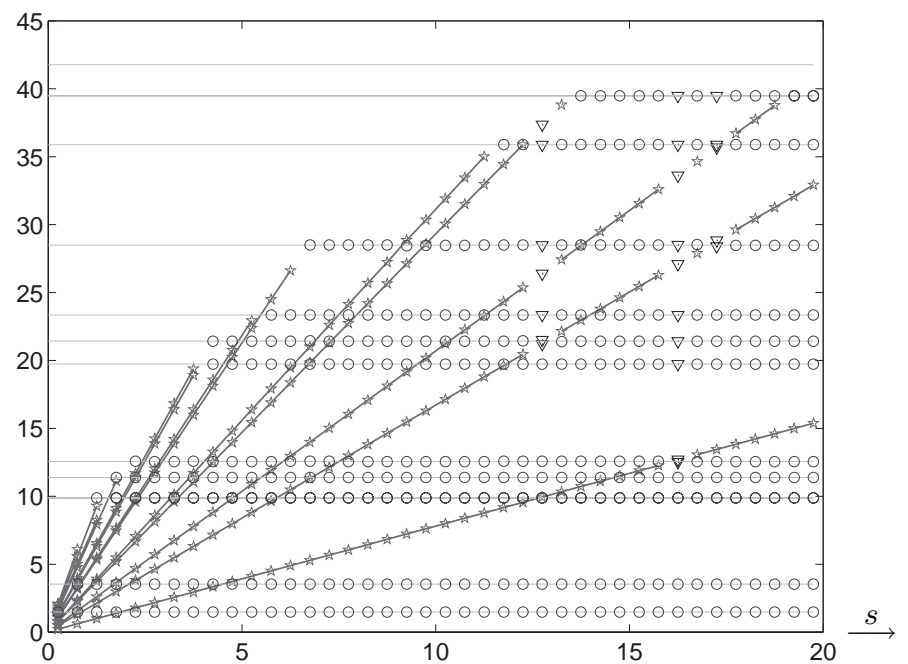

Fig. 9. L-shape, WRM with $\gamma=1$ and $s: 0.25 \rightarrow 20$. Eigenvalues vs parameter $s$.

The results with $\gamma=1$ have a similar appearance to those of the square in Fig.6: Each Maxwell eigenvalue is approximated by an eigenvalue of the discrete problem (almost constant with respect to $s$ ) and conversely the spurious eigenvalues depend linearly on $s$ and escape when $s$ increases - the smallest slope is indeed larger than $\frac{4}{9}$. We note that larger values of $s$ have to be preferred when $\gamma$ increases.

For the $h$-version of finite element method, combining: 
a. The general convergence estimate of eigenvalues (9.6),

b. The best approximation rates of the WRM proved in [28, Th.7.4],

c. The regularity of the first two Maxwell eigenvectors $\mathbf{u}_{1}$ and $\mathbf{u}_{2}$ on this symmetric L-shape (i.e. $\mathbf{u}_{1} \in H^{\frac{2}{3}-\varepsilon}(\Omega), \mathbf{u}_{2} \in H^{\frac{4}{3}-\varepsilon}(\Omega)$ for all $\varepsilon>0$ ),

we obtain the theoretical convergence rates with respect to the number of unknowns $N \simeq h^{-2}$ :

$$
\left|\lambda_{h, 1}-\lambda_{1}\right| \leq C N^{-\left(\gamma-\frac{1}{3}\right)+\varepsilon} \quad \text { and } \quad\left|\lambda_{h, 2}-\lambda_{2}\right| \leq C N^{-\left(\gamma+\frac{1}{3}\right)+\varepsilon},
$$

for example using rectangular elements of degree $p \geq 3$. We stress that the exponent $\gamma-\frac{1}{3}$ is optimal for the first eigenvector and thus the condition $\gamma>\frac{1}{3}$ is necessary to obtain convergence. The second eigenvector is more regular and can be approximated without WRM as was visible on Fig.7, but the convergence rate is improved with the use of the WRM.

\begin{tabular}{|c|c|c|c|c|c|c|c|c|}
\hline & \multicolumn{2}{|c|}{ Neumann } & \multicolumn{2}{|c|}{ WRM $\quad \gamma=0$} & \multicolumn{2}{|c|}{ WRM $\quad \gamma=\frac{1}{2}$} & \multicolumn{2}{|c|}{ WRM $\quad \gamma=1$} \\
\hline$N$ & $\left|\lambda_{1}-\lambda_{h, 1}\right|$ & $\tau$ & $1-\lambda_{h, 1} \mid$ & $\tau$ & $\lambda_{1}-\lambda_{h, 1} \mid$ & $\tau$ & $\left|\lambda_{1}-\lambda_{b_{1}}\right|$ & $\tau$ \\
\hline 42 & 0 & & & & & & & \\
\hline 130 & 0.00861570 & 0.843 & & 0.191 & & 0.231 & 287 & 0.876 \\
\hline 450 & 0.0033 & 0.750 & & 0.040 & 37 & 0.100 & & 0.776 \\
\hline 1666 & 0.00134663 & 0.707 & 3.1077517 & 0.013 & 2.2467554 & 0.083 & 0.0597067 & 0.731 \\
\hline$N$ & $\left|\lambda_{2}-\lambda_{h, 2}\right|$ & $\tau$ & 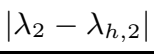 & $\tau$ & | & $\tau$ & $\left|\lambda_{2}-\lambda_{h, 2}\right|$ & $\tau$ \\
\hline 42 & 0 & & & & 02 & & 20 & \\
\hline 130 & 0.0011 & 2.277 & & 1.043 & & 1.093 & 193 & 1.657 \\
\hline 450 & 0.00010186 & 1.944 & 0.0392901 & 0.798 & 0.0271478 & 0.853 & 0.0021616 & 1.446 \\
\hline 1666 & 0.00001117 & 1.689 & 0.0152519 & 0.723 & 0.0097170 & 0.785 & 0.0003549 & 1.380 \\
\hline
\end{tabular}

Table 2. L-shape, uniform grids, $p=2$. Errors and convergence rates

We illustrate the error estimates (9.7) by choosing $\mathbb{Q}_{p}$ elements on a sequence of uniform grids, starting with a very simple grid containing only 3 squares which are then repeatedly sub-divided into four. In Tables 2 and 3, we show errors and convergence rates for the computation of the first two Maxwell eigenvalues. We choose $s=100$ to avoid the spurious eigenvalues. With weight exponent $\gamma=1$, we see the expected convergence rates $\tau=2 / 3$ for the first eigenvalue and $\tau=4 / 3$ for the second eigenvalue.

Other numerical results are provided in [30], both for the above L-shaped domain and for another L-shaped domain with curved sides. We show in particular how the error decreases when a geometrically refined mesh is used together with high degree $p$ : With the degree $p$ equal to the number of layers of the mesh, an exponential rate of convergence is obtained, see also [32]. 


\begin{tabular}{|r|c|c|c|c|c|c|c|c|}
\hline & \multicolumn{2}{|c|}{ Neumann } & WRM $\gamma=0$ & WRM & $\gamma=\frac{1}{2}$ & \multicolumn{2}{|c|}{ WRM } & $\gamma=1$ \\
\hline \hline$N$ & $\left|\lambda_{1}-\lambda_{h, 1}\right|$ & $\tau$ & $\left|\lambda_{1}-\lambda_{h, 1}\right|$ & $\tau$ & $\left|\lambda_{1}-\lambda_{h, 1}\right|$ & $\tau$ & $\left|\lambda_{1}-\lambda_{h, 1}\right|$ & $\tau$ \\
\hline 450 & 0.00084498 & & 3.1789407 & & 2.1954444 & & 0.0374398 & \\
1666 & 0.00033527 & 0.706 & 3.1149073 & 0.016 & 1.9323080 & 0.098 & 0.0147321 & 0.713 \\
6402 & 0.00013226 & 0.691 & 3.0899818 & 0.006 & 1.6883397 & 0.100 & 0.0057792 & 0.695 \\
25090 & 0.00005169 & 0.688 & 3.0801659 & 0.002 & 1.4581948 & 0.107 & 0.0022747 & 0.683 \\
\hline$N$ & $\left|\lambda_{2}-\lambda_{h, 2}\right|$ & $\tau$ & $\left|\lambda_{2}-\lambda_{h, 2}\right|$ & $\tau$ & $\left|\lambda_{2}-\lambda_{h, 2}\right|$ & $\tau$ & $\left|\lambda_{2}-\lambda_{h, 2}\right|$ & $\tau$ \\
\hline 450 & 0.00000215 & & 0.0478032 & & 0.0176105 & & 0.0001714 & \\
1666 & 0.00000034 & 1.408 & 0.0185193 & 0.724 & 0.0063407 & 0.780 & 0.0000305 & 1.318 \\
6402 & 0.00000005 & 1.372 & 0.0072806 & 0.694 & 0.0021963 & 0.788 & 0.0000050 & 1.335 \\
25090 & 0.00000001 & 1.345 & 0.0028786 & 0.679 & 0.0007433 & 0.793 & 0.0000008 & 1.338 \\
\hline
\end{tabular}

Table 3. L-shape, uniform grids, $p=8$. Errors and convergence rates

Another situation where the WRM turns out to be very efficient is the problem with an impedance boundary condition. In [29] we showed numerical results for a boundary penalization method where the perfect conductor boundary condition on an L-shaped domain is replaced by an impedance-like condition. According to the density result in Theorem 2.1 for $W_{N}$, the standard regularization method leads to a convergent Galerkin approximation even in the presence of non- $H^{1}$ singularities. In the experiments in [29], one sees convergence, but the convergence rate is extremely low, so that the method is not practical. By using the WRM with $\gamma=1$ on the L-shape domain, one observes the same good convergence properties as reported here for the perfect conductor boundary condition. The boundary penalization, when combined with the weighted regularization, can therefore be employed efficiently as an alternative to the use of essential boundary conditions.

\section{F 3D computations}

To test our code (the finite element library MÉLINA [54]) and our weighted regularization method for three-dimensional domains, we first performed experiments with the thick L-shaped domain formed by taking the tensor product of the 2D L-shape with the interval $(0,1)$ and obtained the expected behaviour of computed eigenvalues. These experiments will be reported elsewhere.

Instead, we shall report on computations using the archetypical three-dimensional corner:

$$
\Omega=(-1,1)^{3} \backslash(-1,0)^{3} .
$$

We refer to this as the Fichera corner, in honour of the fact that FICHERA was the first to estimate the lowest Laplace-Dirichlet corner exponent at the corner $\mathbf{c}=0$ : in [41] the estimate $0.4335<\lambda_{\mathrm{c}}^{1, \text { Dir }}<0.4645$ is given. In [60], a computation by a boundary element method yields the approximate value $\lambda_{\mathrm{c}}^{1, \text { Dir }} \simeq 0.45418$. 
Let us recall, cf Table 1, that the set of Maxwell corner exponents is the union of the Dirichlet-Laplace exponents minus 1 and the Neumann-Laplace. Thomas APEL provided us with the following numerical approximation of the first Laplace exponents for the Fichera corner (computed with the method of [3]), see Table 4. Thus

\begin{tabular}{|c|c|}
\hline Dirichlet & Neumann \\
\hline 0.45418 & 0.00000 \\
1.23088 & 0.84001 \\
1.23090 & 0.84002 \\
1.78432 & 1.20637 \\
2.11766 & 1.80618 \\
2.11768 & 1.80619 \\
\hline
\end{tabular}

Table 4. Fichera corner. Laplacian corner exponents

the first Maxwell exponents are $-0.5459,0.2309,0.7843,0.8400$, etc...

The domain $\Omega$ has three non-convex edges $\mathbf{e}_{1}, \mathbf{e}_{2}$ and $\mathbf{e}_{3}$ which coincide with the interval $(-1,0)$ in each coordinate axis. As distance function $d_{0}$, we can choose the minimum of the distance functions $d_{j}$ to each edge $\mathbf{e}_{j}$, or take the product $d_{1} d_{2} d_{3}$ divided by $\rho^{2}$ where $\rho$ is the distance to 0 .

We performed computations with a tensor mesh (each side of the 7 cubes which form $\Omega$ is divided into 3 subintervals according to the ratios $\left.0, \frac{1}{64}, \frac{1}{2}, 1\right)$ and $\mathbb{Q}_{4}$

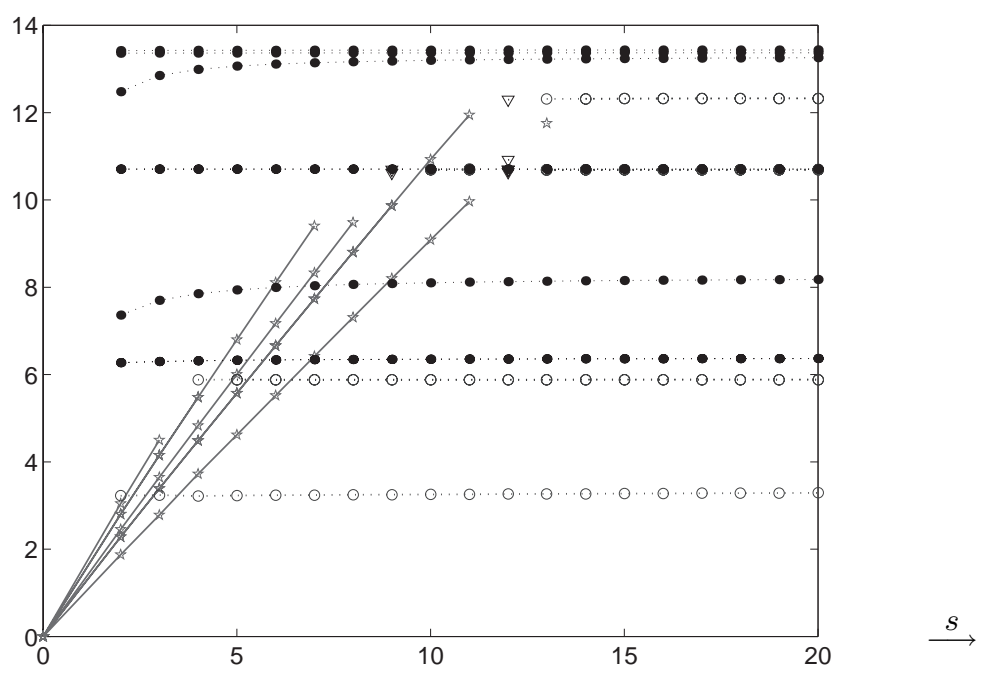

Fig. 10. Fichera corner, WRM with $\gamma=1$ and $s: 1 \rightarrow 20$. Parameter $s$ vs eigenvalues. 
elements are used (which produces 41691 degrees of freedom for the Maxwell system). In Fig. 10 we plot the 8 lowest computed eigenvalues obtained with the WRM and $\gamma=1$ (circles, pentagrams and triangles according to the conventions in $\S 7 . \mathrm{B}$ ), and also the results obtained without weight $(\gamma=0)$, represented on the same graph as dots $\bullet$. Both families (Maxwell and spurious) are connected by lines. Reference values for the exact Maxwell eigenvalues are not available.

\section{Comparisons}

\section{A Miscellaneous penalized formulations}

Two more methods for plane domains using "penalized" formulations (i.e. in our words "regularization") are described in [17] together with numerical experiments. One of them consists of the use of the piecewise linear nonconforming elements of CROUZEIX-RAVIART [33] in the plain regularized bilinear form (7.1).

The other method can be expressed as a three field method involving the electric field and its (scalar) curl and divergence. It is similar to that of [6] and has been partially analyzed in [44]. This method is equivalent to a plain regularized formulation with projection. Given a mesh $\mathcal{T}_{h}$, let $V_{h}, M_{h}$ and $Q_{h}$ be the following three spaces:

$$
\begin{aligned}
V_{h} & =\left\{\mathbf{v}_{h} \in X_{N}, \quad \mathbf{v}_{h} \text { piecewise } \mathbb{Q}_{2}\right\} \\
M_{h} & =\left\{\mu_{h} \in L_{0}^{2}(\Omega), \quad \mu_{h} \text { piecewise } \mathbb{P}_{1}\right\} \\
Q_{h} & =\left\{q_{h} \in L^{2}(\Omega), \quad q_{h} \text { piecewise } \mathbb{P}_{1}\right\}
\end{aligned}
$$

Here $L_{0}^{2}(\Omega)$ denotes the space of $L^{2}$ functions with zero mean value over $\Omega$. Let $P_{1}$ and $P_{1,0}$ denote the $L^{2}$ projections over $Q_{h}$ and $M_{h}$, respectively. Then the three field method is equivalent to solve

$$
\mathbf{u}_{h} \in \mathfrak{X}_{h}, \quad \forall \mathbf{v}_{h} \in \mathfrak{X}_{h}, \quad a\left(\mathbf{u}_{h}, \mathbf{v}_{h}\right)=\omega_{h}^{2} \int_{\Omega} \mathbf{u}_{h} \cdot \mathbf{v}_{h} \mathrm{~d} \mathbf{x}
$$

with the space $\mathfrak{X}_{h}=V_{h}$ and the bilinear form $a=a_{s}^{h}$

$$
a_{s}^{h}(\mathbf{u}, \mathbf{v})=\int_{\Omega} P_{1,0}(\operatorname{curl} \mathbf{u}) P_{1,0}(\operatorname{curl} \mathbf{v}) \mathrm{d} \mathbf{x}+s \int_{\Omega} P_{1}(\operatorname{div} \mathbf{u}) P_{1}(\operatorname{div} \mathbf{v}) \mathrm{d} \mathbf{x} .
$$

\section{B Numerical results on the L-shape}

it is interesting to compare the results in [17] with what can be obtained by the weighted regularization. The L-shape is now $(0, \pi)^{2} \backslash(0, \pi / 2)^{2}$. We add to the results from [17, Table II] reference values (the first twelve eigenvalues of the Neumann scalar problem) and two series of computations made by the WRM with $\mathbb{Q}_{2}$ and $\mathbb{Q}_{4}$ elements.

In Table 5, columns 2, 3 and 4 are taken from [17]: $\mathbb{P}_{1}^{C R}$ refers to the CROUZEIXRAVIART elements, whereas $\mathbb{Q}_{2}^{P}$ refers to the nodal biquadratic elements with projection. The value of $s$ is 100 for both, and 12.5 for the WRM. In the WRM series 


\begin{tabular}{|r|r|c|r|r|r|}
\hline $\begin{array}{r}\text { Neumann } \\
\text { 1581 d.o.f. }\end{array}$ & $\begin{array}{r}\text { Edge } \mathbf{P}_{1} \\
910 \text { d.o.f. }\end{array}$ & $\begin{array}{r}\mathbb{P}_{1}^{C R} \\
966 \text { d.o.f. }\end{array}$ & $\begin{array}{r}\mathbb{Q}_{2}^{P} \\
962 \text { d.o.f. }\end{array}$ & $\begin{array}{r}\text { WRM } \mathbb{Q}_{2} \\
1050 \text { d.o.f. }\end{array}$ & $\begin{array}{r}\text { WRM } \mathbb{Q}_{4} \\
1050 \text { d.o.f. }\end{array}$ \\
\hline 0.59805 & 0.59179 & 0.61595 & 0.60510 & 0.61216 & 0.60166 \\
1.43229 & 1.43232 & 1.45070 & 1.43314 & 1.43265 & 1.43232 \\
4.00000 & 4.00554 & 3.93313 & 4.00047 & 4.00180 & 4.00000 \\
4.00000 & 4.00554 & 4.01422 & 4.00049 & 4.00182 & 4.00000 \\
4.61598 & 4.61320 & 4.60196 & 4.62364 & 4.62132 & 4.61600 \\
5.09540 & 5.06733 & 5.07813 & 5.13854 & 5.10554 & 5.09633 \\
8.00000 & 7.95513 & 7.90668 & 8.08411 & 8.03128 & 8.00008 \\
8.68312 & 8.64737 & 8.71656 & 8.76070 & 8.70613 & 8.67862 \\
9.46112 & 9.48166 & 9.57140 & 9.48655 & 9.49236 & 9.46145 \\
11.54689 & 11.4261 & 11.5640 & 11.8268 & 11.64367 & 11.54926 \\
14.54106 & 14.4486 & 14.5241 & 14.8680 & 14.72710 & 14.54433 \\
16.00000 & 16.0862 & 15.6111 & 16.0254 & 16.10483 & 16.00022 \\
\hline \multicolumn{7}{|c|}{ Number of computed zeros } \\
\hline 1
\end{tabular}

Table 5. L-shape. First 12 eigenvalues by different methods

we have removed the spurious values which were present: 10.4818282 for the degree 2 and 8.9545298 for the degree 4 . Such a spurious value is easily recognized by its small ratio $\tau\left(\mathbf{u}_{h}\right)$, see (7.2). Another way to trace them is to compute for two different value of $s$.

We can note that in the series $\mathbb{P}_{1}^{C R}$ and $\mathbb{Q}_{2}^{P}$ it often happens that a computed eigenvalue is below its exact counterpart. For the WRM, this never happens below $s \sigma_{0}$, with $\sigma_{0}$ the lower limit of the spurious Laplacian $L_{\gamma}^{\text {Dir }}, \operatorname{cf}$ Cor.9.7, (here $\gamma=1$, $\sigma_{0}=\frac{4}{9}$ and $s \sigma_{0} \simeq 5.5555$ ). It may (seldom) happen above (the value 8.67862 in the degree 4 series).

\section{C The benchmark page}

As a step towards further comparison of the performance of different numerical methods for the Maxwell eigenvalue problem, a web page has been created containing the results of the $2 \mathrm{D}$ and $3 \mathrm{D}$ computations presented in this section. At

$$
\text { http://www.maths.univ-rennes1.fr/ dauge/benchmax.html }
$$

one can find detailed descriptions of the geometries of the model boundary value and eigenvalue problems as well as the numerical results of computations using the weighted regularization method. In addition to the L-shaped domain and the Fichera corner, there are results in 2D for a square with a crack, a curved rectangle, a curved $\mathrm{L}$ and a transmission problem on a square composed of four subdomains with different permittivities, and in 3D for the "thick L". 
Contributions to this web-page are invited in the form of numerical results for the same benchmark problems. In particular, it will be very interesting to compare results obtained with the weighted regularization method and nodal finite elements to results obtained with various other variational formulations and edge elements.

\section{References}

1. S. AdAm, P. Arbenz, R. Geus. Eigenvalue solvers for electromagnetic fields in cavities. Technical Report 275, Institute of Scientific Computing, ETH Zürich 1997.

2. C. Amrouche, C. Bernardi, M. Dauge, V. Girault. Vector potentials in threedimensional nonsmooth domains. Math. Meth. Appl. Sci. 21 (1998) 823-864.

3. T. Apel, V. Mehrmann, D. Watkins. Structured eigenvalue methods for the computation of corner singularities in 3D anisotropic elastic structures. Comput. Methods Appl. Mech. Engrg. 191 (2002) 4459-4473.

4. F. Assous, P. Ciarlet, E. SonnendrüCKer. Résolution des équations de Maxwell dans un domaine avec un coin rentrant. C. R. Acad. Sc. Paris, Série I 323 (1996) 203208.

5. I. BABUŠKA, J. E. OSBORN. Finite element-Galerkin approximation of the eigenvalues and eigenvectors of selfadjoint problems. Math. Comp. 52(186) (1989) 275-297.

6. K.-J. Bathe, C. Nitikitpaiboon, X. WAng. A mixed displacement-based finite element formulation for acoustic fluid-structure interaction. Comput. \& Structures 56(23) (1995) 225-237.

7. F. Ben Belgacem, C. Bernardi, M. Costabel, M. Dauge. Un résultat de densité pour les équations de Maxwell. C. R. Acad. Sci. Paris Sér. I Math. 324(6) (1997) 731736.

8. A. N. Bespalov. Finite element method for the eigenmode problem of a RF cavity resonator. Soviet J. Numer. Anal. Math. Modelling 3(3) (1988) 163-178.

9. M. Birman, M. SOlOMYAK. $L^{2}$-theory of the Maxwell operator in arbitrary domains. Russ. Math. Surv. 42 (6) (1987) 75-96.

10. M. Birman, M. SOlOMYAK. On the main singularities of the electric component of the electro-magnetic field in regions with screens. St. Petersbg. Math. J. 5 (1) (1993) 125-139.

11. D. BofFI. Fortin operator and discrete compactness for edge elements. Numer. Math. 87(2) (2000) 229-246.

12. D. Boffi. A note on the de Rham complex and a discrete compactness property. Appl. Math. Lett. 14(1) (2001) 33-38.

13. D. Boffi, F. BREZzI, L. GAStALDI. On the convergence of eigenvalues for mixed formulations. Ann. Scuola Norm. Sup. Pisa Cl. Sci. (4) 25(1-2) (1997) 131-154 (1998). Dedicated to Ennio De Giorgi.

14. D. Boffi, F. BREzzI, L. GAStALDI. On the problem of spurious eigenvalues in the approximation of linear elliptic problems in mixed form. Math. Comp. 69(229) (2000) 121-140.

15. D. Boffi, L. Demkowicz, M. Costabel. Discrete compctness for $p$ and $h p 2 \mathrm{~d}$ edge finite elements. TICAM Report 02-21, Université de Bordeaux 1, 2002.

16. D. Boffi, R. G. DuRAn, L. GAstaldi. A remark on spurious eigenvalues in a square. Appl. Math. Lett. 12(3) (1999) 107-114.

17. D. Boffi, M. FARINA, L. Gastaldi. On the approximation of Maxwell's eigenproblem in general 2D domains. Comput. \& Structures 79 (2001) 1089-1096. 
18. D. Boffi, P. Fernandes, L. Gastaldi, I. Perugia. Computational models of electromagnetic resonators: analysis of edge element approximation. SIAM J. Numer. Anal. 36 (1999) 1264-1290.

19. A.-S. Bonnet-Ben Dhia, C. Hazard, S. Lohrengel. A singular field method for the solution of Maxwell's equations in polyhedral domains. SIAM J. Appl. Math. 59(6) (1999) 2028-2044 (electronic).

20. S. CaOrsi, P. Fernandes, M. Raffetto. On the convergence of Galerkin finite element approximations of electromagnetic eigenproblems. SIAM J. Numer. Anal. 38(2) (2000) 580-607 (electronic).

21. S. CAorsi, P. Fernandes, M. Raffetto. Spurious-free approximations of electromagnetic eigenproblems by means of Nedelec-type elements. M2AN Math. Model. Numer. Anal. 35(2) (2001) 331-354.

22. P. Ciarlet, Jr., C. Hazard, S. Lohrengel. Les équations de Maxwell dans un polyèdre : un résultat de densité. C. R. Acad. Sc. Paris, Série I Math. 326(11) (1998) 1305-1310.

23. M. Costabel. A coercive bilinear form for Maxwell's equations. J. Math. Anal. Appl. 157 (2) (1991) 527-541.

24. M. Costabel, M. Dauge. Espaces fonctionnels Maxwell: Les gentils, les méchants et les singularités. On line publication (Dec. 1998): http: //www. maths.univ-rennes1.fr/ dauge.

25. M. Costabel, M. Dauge. Un résultat de densité pour les équations de Maxwell régularisées dans un domaine lipschitzien. C. R. Acad. Sc. Paris, Série I 327 (1998) 849-854.

26. M. Costabel, M. Dauge. Maxwell and Lamé eigenvalues on polyhedra. Math. Meth. Appl. Sci. 22 (1999) 243-258.

27. M. Costabel, M. Dauge. Singularities of electromagnetic fields in polyhedral domains. Arch. Rational Mech. Anal. 151(3) (2000) 221-276.

28. M. Costabel, M. Dauge. Weighted regularization of Maxwell equations in polyhedral domains. Numer. Math. 93 (2) (2002) 239-277.

29. M. Costabel, M. Dauge, D. Martin. Numerical investigation of a boundary penalization method for Maxwell equations. In P. NeITTAANMÄKI, T. TIIHONEN, P. TARVAINEN, editors, Proceedings of the 3rd European Conference on Numerical Mathematics and Advanced Applications, pages 214-221. World Scientific, Singapore 2000.

30. M. Costabel, M. Dauge, D. Martin, G. Vial. Weighted regularization of Maxwell equations - computations in curvilinear polygons. In Proceedings of the 4th European Conference on Numerical Mathematics and Advanced Applications. Springer 2002.

31. M. Costabel, M. Dauge, S. Nicaise. Singularities of Maxwell interface problems. M2AN Math. Model. Numer. Anal. 33(3) (1999) 627-649.

32. M. Costabel, M. Dauge, C. Schwab. Exponential convergence of the $h p$-FEM for the weighted regularization of Maxwell equations in polygonal domains. In preparation.

33. M. Crouzeix, P.-A. RaviarT. Conforming and nonconforming finite element methods for solving the stationary Stokes equations. I. Rev. Française Automat. Informat. Recherche Opérationnelle Sér. Rouge 7(R-3) (1973) 33-75.

34. M. DAUGE. "Simple" corner-edge asymptotics. On line publication (Dec. 2000): http: //www.maths.univ-rennes1.fr/ dauge.

35. M. DAUge. Elliptic Boundary Value Problems in Corner Domains - Smoothness and Asymptotics of Solutions. Lecture Notes in Mathematics, Vol. 1341. Springer-Verlag, Berlin 1988.

36. M. DAUGE. Neumann and mixed problems on curvilinear polyhedra. Integral Equations Oper. Theory. 15 (1992) 227-261. 
37. M. DAUGE. Singularities of corner problems and problems of corner singularities. In Actes du 30ème Congrès d'Analyse Numérique: CANum '98 (Arles, 1998), pages 19-40 (electronic). Soc. Math. Appl. Indust., Paris 1999.

38. L. Demkowicz, P. MonK. Discrete compactness and the approximation of Maxwell's equations in $\mathbb{R}^{3}$. Math. Comp. 70 (2001) 507-523.

39. J. DesclouX. Essential numerical range of an operator with respect to a coercive form and the approximation of its spectrum by the Galerkin method. SIAM J. Numer. Anal. 18(6) (1981) 1128-1133.

40. J. DesclouX, N. Nassif, J. Rappaz. On spectral approximation. I. The problem of convergence. RAIRO Anal. Numér. 12(2) (1978) 97-112, iii.

41. G. FicherA. Comportamento asintotico del campo elettrico e della densità elettrica in prossimità dei punti singolari della superficie conduttore. Rend. Sem. Mat. Univ. e Politec. Torino 32 (1973/74) 111-143.

42. N. Filonov. Système de Maxwell dans des domaines singuliers. Thesis, Université de Bordeaux 1, 1996.

43. E. GARCiA. Résolution des équation de Maxwell instationnaires dans des domaine non convexe, la méthode du complément singulier. Thèse, Université Pierre et Marie Curie 2002.

44. L. Gastaldi. Mixed finite element methods in fluid structure systems. Numer. Math. 74(2) (1996) 153-176.

45. V. Girault, P. Raviart. Finite Element Methods for the Navier-Stokes Equations, Theory and Algorithms. Springer series in Computational Mathematics, 5. SpringerVerlag, Berlin 1986.

46. P. GRISVARD. Boundary Value Problems in Non-Smooth Domains. Pitman, London 1985.

47. C. HAZARD. Numerical simulation of corner singularities: a paradox in Maxwell-like problems. C. R. Mecanique 330 (2002) 57-68.

48. C. Hazard, S. Lohrengel. A singular field method for Maxwell's equations: Numerical aspects in two dimensions. SIAM J. Numer. Anal. (2002) To appear.

49. R. Hiptmair. Finite elements in computational electromagnetism. Acta Numerica (2002) 237-339.

50. F. KIKUCHI. Mixed and penalty formulations for finite element analysis of an eigenvalue problem in electromagnetism. In Proceedings of the first world congress on computational mechanics (Austin, Tex., 1986), volume 64, pages 509-521 1987.

51. F. KIKUCHI. On a discrete compactness property for the Nédélec finite elements. J. Fac. Sci. Univ. Tokyo Sect. IA Math. 36(3) (1989) 479-490.

52. V. A. Kondrat'EV. Boundary-value problems for elliptic equations in domains with conical or angular points. Trans. Moscow Math. Soc. 16 (1967) 227-313.

53. R. LEIS. Zur Theorie elektromagnetischer Schwingungen in anisotropen inhomogenen Medien. Math. Z. 106 (1968) 213-224.

54. D. Martin. Mélina. On line documentation: http: //www. maths. univ-rennes1.fr/ dmartin.

55. S. A. Nazarov, B. A. Plamenevs KiI. Elliptic Problems in Domains with Piecewise Smooth Boundaries. Expositions in Mathematics 13. Walter de Gruyter, Berlin 1994.

56. J.-C. NÉDÉLEC. Mixed finite elements in $\mathbb{R}^{3}$. Numer. Math. 35 (1980) 315-341.

57. J.-C. NÉDÉLEC. A new family of mixed finite elements in $\mathbb{R}^{3}$. Numer. Math. 50(1) (1986) 57-81.

58. K. PREISS, O. BIRó, I. TICAR. Gauged current vector potential and reentrant corners in the FEM analysis of 3D eddy currents. IEEE Transactions on Magnetics 36(4) (2000) 840-843. 
59. P.-A. Raviart, J. M. Thomas. Primal hybrid finite element methods for 2 nd order elliptic equations. Math. Comp. 31(138) (1977) 391-413.

60. H. Schmitz, K. VolK, W. Wendland. Three-dimensional singularities of elastic fields near vertices. Numer. Methods Partial Differential Equations 9(3) (1993) 323337.

61. C. SchwaB. p- and hp-finite element methods. Theory and applications in solid and fluid mechanics. The Clarendon Press Oxford University Press, New York 1998.

62. M. SuRI, C. Xenophontos. Reliability of an $h p$ algorithm for buckling analysis. Proceedings of IASS-IACM 2000, Fourth International Colloquium on Computation of Shell and Spatial Structures, 2000 (CD-Rom).

63. H. VANDEVEN. On the eigenvalues of second-order spectral differentiation operators. Comput. Methods Appl. Mech. Engrg. 80(1-3) (1990) 313-318. Spectral and high order methods for partial differential equations (Como, 1989). 